\title{
DESIGN OF SINGLET FISSION CHROMOPHORES WITH CYCLIC (ALKYL)(AMINO) CARBENE BUILDING BLOCKS
}

by

\author{
Achini Japahuge
}

A thesis submitted to the Faculty of Graduate and Postdoctoral Affairs in partial fulfilment of the requirements of the degree of

Master of Science

In

Chemistry

Carleton University

Ottawa, Ontario

(C) 2019

Achini Japahuge 


\begin{abstract}
This is a theoretical chemistry study to design singlet fission chromophores. Quantum chemistry methods MRSF-TDDFT and NEVPT2 are used to design chromophores with the building blocks of cyclic (alkyl)(amino)carbenes (CAACs). CAAC dimers with $\mathrm{C}_{2}, \mathrm{C}_{4}$ and $p$-phenylene spacers are considered. The substitutions with trifluoromethyls and fluorine atoms at the $\alpha$ position are investigated. The electronegative substituents enhance the $\pi$ accepting capability of the $\alpha \mathrm{C}$, while maintaining it as a quaternary $\mathrm{C}$ atom. The phenylene-connected dimers with the two substitutions are identified as promising candidates for the singlet fission chromophores. The cylindrically symmetric $\mathrm{C}_{2}$ and $\mathrm{C}_{4}$ spacers allow for substantial structural reorganizations in the $S_{0}$-to- $S_{1}$ and $S_{0}$-to- $T_{1}$ excitations. Although the two substituted dimers with the $\mathrm{C}_{4}$ spacer satisfy (or very close to satisfy) the primary thermodynamics criterion for the singlet fission, the significant structural reorganizations result in high barriers so that the fission is kinetically unfavorable.
\end{abstract}




\section{Acknowledgement}

First of all, I would like to express my sincere gratitude to my supervisor Dr. Tao Zeng for his patient and immense support during my research. I consider myself lucky to work with him and share his knowledge in academic and professional life. My project couldn't have a successful one without him.

I am also grateful to Dr. Seunghoon Lee at Seoul National University and Professor Cheol Ho Choi at Kyungpook National University. They developed the MRSFTDDFT method that is used to optimize the structures of the designed molecules and calculate their vibrational frequencies.

I would like to thank you all the members who were working with me in the lab, for their support during this time. I would also like to thank you Prof. Robert Crutchley head of the department, who guided us during the seminar sessions that helped me to improve my presentation skills. Also, my heartfelt gratitude goes to Chantelle Gravelle for her immense support on behalf of the administration of the Department of Chemistry.

Further, A very special gratitude goes to the team of Calcul Québec and Compute Canada who provided technical support in terms of calculations and data collecting.

Finally, I am grateful for my family members in Sri Lanka, who have always been supporting me during this time. Thank you all for your encouragement! 


\section{Table of Contents}

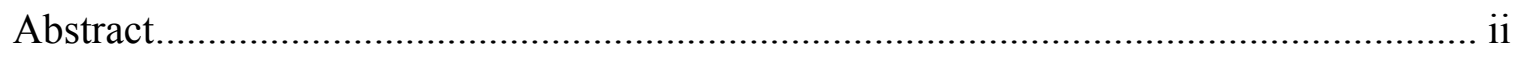

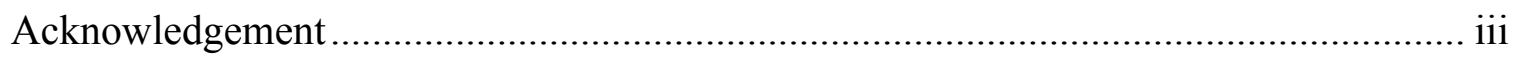

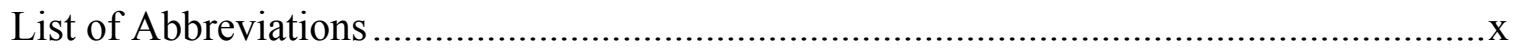

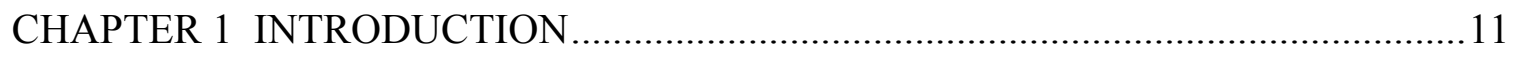

Section 1.1 Singlet Fission, an Optoelectronics Phenomenon ................................. 11

Section 1.2 Electronic Structure Description of Singlet Fission ............................... 13

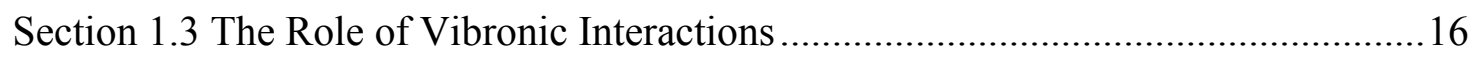

Section 1.4 Searching for Singlet Fission Chromophores .......................................18

Section 1.5 Alternant Hydrocarbons and Diradicals; The Two Limits .......................20

Section 1.6 A Unified Diradical Character View of the SF Chromophores .................22

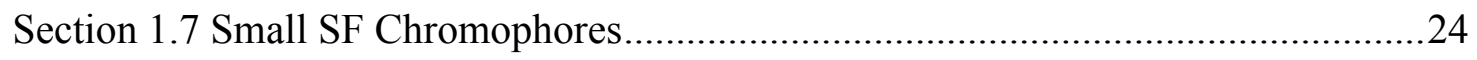

Section 1.8 Summary of Promising Theoretical Design .........................................28

Section 1.9 Design of SF Chromophores with Cyclic (Alkyl)(Amino) Carbene

Building Blocks, the Motivation and Objective of This Work ................................29

Section 1.10 Organization of the Rest of the Thesis ............................................. 31

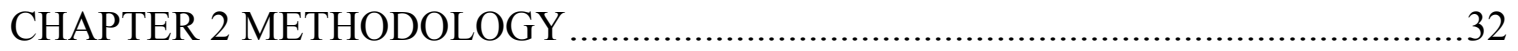

Section 2.1 MRSF-TDDFT Method for Electronic Structure Correlation ....................32

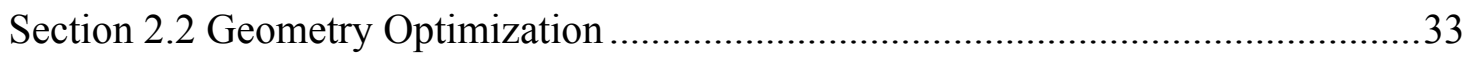

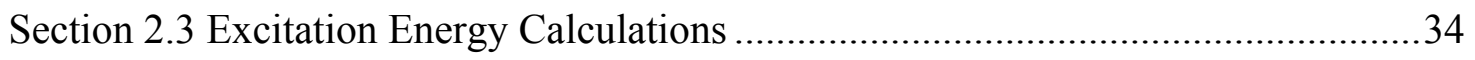


Section 3.1 The Fluorination and Trifluoromethylation...............................................36

Section 3.2 CAAC Dimers with a $\mathrm{C}_{2}$ Spacer ………...................................................

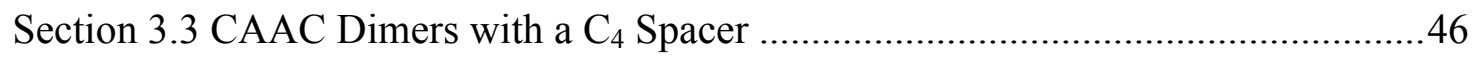

Section 3.4 CAAC Dimers with a Para-Phenylene Spacer .........................................54

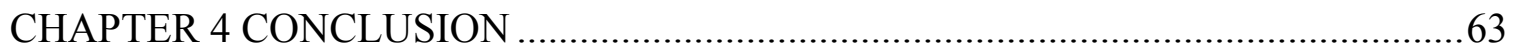

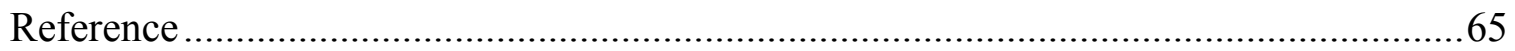




\section{List of Figures}

Figure 1. A comparison of photoelectric conversions without (upper panel) and with ( lower panel) singlet fission. There are common steps in both panels : (a) photoexcitation, (b) exciton migration, and (c) charge separation between chromophore and acceptor. Steps (d) and (e) are radiationless and radiative decays of singlet exciton. .11

Figure 2. A schematic description of SF. The vertical arrows represent electronic spin. In the state labels, e stands for the HOMO-to-LUMO singlet exciton, $t$ is the triplet exciton, $g$ is the ground state, $c$ is the cationic state, and $a$ is the anionic state. A vertical dashed line is added to the $t+t$ state to emphasize that the two triplets are independent of each other.

Figure 3. (a) Vibrational wave packets on different potential energy surfaces and their transitions to different states in SF; evolutions of diabatic populations in a SF process from dynamics simulations that consider (b) vibronic interaction and (c) only the electronic Hamiltonian. In panel (c), only the ME state population is plotted to show the Rabi oscillation. Panels (b) and (c) are adapted from Ref. 24.

Figure 4. Characteristic energy levels of the closed- and open-shell parental structures (at the left and the right end), and their changes when the structures are perturbed towards singlet fission chromophores (in the middle).

Figure 5. Weakening of the covalent interaction between two methylene radicals in forming the $\pi$ bond of ethylene when they contain acceptor (A) and donor (D) substituents. .26 
Figure 6. (a) and (b) the $\sigma$ HOMO and $\pi$ LUMO of CAAC in its singlet ground state; (c) the $\pi$ LUMO of CAAC $(\mathrm{F})$. .36

Figure 7 . The optimized structures of the (a) $S_{0}$, (b) $T_{1}$, and (c) $S_{1}$ states of $(\mathrm{CAAC})_{2}-\mathrm{C}_{2}$; (d)-(g) frontier orbitals of the structures of the denoted states; (h)-(i) typical resonance structures of the three states. Some important structural parameters are shown in panels (a)-(c), where the bond lengths are given in $\AA$ and the four atoms that define the dihedral angles are highlighted by red circles. In panel (i), only one set of resonance structures are shown for the $S_{1}$ state. There is another set which includes the symmetry images of the shown structures.

Figure 8 . The optimized structures of the (a) $S_{0}$, (b) $T_{1}$, and (c) $S_{1}$ states of $(\mathrm{CAAC})_{2}-\mathrm{C}_{4}$; (d)-(g) frontier orbitals of the structures of the denoted states; (h)-(j) typical resonance structures of the three states. Some important structural parameters are shown in panels (a)-(c), where the bond lengths are given in $\AA$ and the four atoms that define the dihedral angles are highlighted by red circles. In panel (j), only one set of resonance structures are shown for the $S_{l}$ state. There is another set which includes the symmetry images of the shown structures.

Figure 9. (a) The two singly occupied molecular orbitals $\mathrm{SOMO}_{1}$ and $\mathrm{SOMO}_{2}$ in the $T_{1}$ state of $(\mathrm{CAAC})_{2}-\mathrm{C}_{4}$ and (b) the corresponding localized orbitals obtained from $1 / \sqrt{ } 2\left(\mathrm{SOMO}_{1} \pm \mathrm{SOMO}_{2}\right)$, which are perpendicular $\pi$ orbitals...............................52

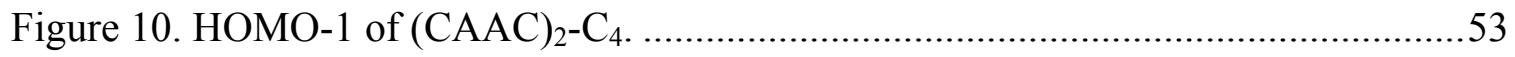

Figure 11. The optimized structures of the (a) $S_{0}$, (b) $T_{1}$, and (c) $S_{1}$ states of $(\mathrm{CAAC}(\mathrm{F}))_{2}$ - $^{-}$ $\mathrm{Ph}$; (d)-(f) frontier orbitals of the structures of the $S_{0}$ state; (g)-(i) typical resonance structures of the three states. Some important structural parameters are shown in 
panels (a)-(c), where the bond lengths are given in $\AA$ and the four atoms that define the dihedral angles are highlighted by red circles. In panel (i), only some resonance structures are shown for the $S_{1}$ state. The others include those with the + and charges being swapped and those with the symmetry images. .55

Figure 12. Localized orbitals $(\mathrm{CAAC}(\mathrm{F}))_{2}-\mathrm{Ph}$ obtained from $1 / \sqrt{2}(\mathrm{HOMO} \pm \mathrm{LUMO}) \ldots .58$

Figure 13. Hydrogen bonds of $\mathrm{F}$ atoms in (a) $(\mathrm{CAAC}(\mathrm{F}))_{2}-\mathrm{Ph}$ and (b) $(\mathrm{CAAC}(\mathrm{TFM}))_{2}-$ $\mathrm{Ph}$. The highlighted internuclear distances are given in $\AA$. The red dashed lines indicate $\mathrm{F} \cdots \mathrm{H}$ hydrogen bonds. .58

Figure 14. A representative structure (a) obtained during the optimization of the $S_{1}$ structure of (CAAC) $)_{2}-\mathrm{Ph}$ and its (b) HOMO and (c) LUMO .60 


\section{List of Tables}

Table 1. Excitation energies (in eV) of theoreticallly designed promising SF

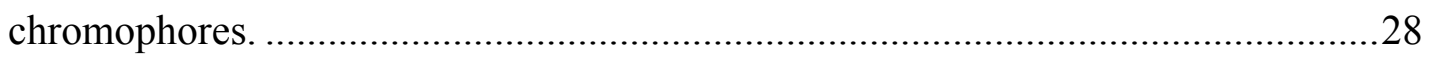

Table 2. Calculated results for the three C2-connected dimers. All energies are given in

$\mathrm{eV}$.

Table 3. Calculated results for the three C4-connected dimers. All energies are given in $\mathrm{eV}$.

Table 4. Calculated results for the two substituted Ph-connected dimers. All energies are given in $\mathrm{eV}$ .56 


\section{List of Abbreviations}

\begin{tabular}{|c|c|}
\hline CAAC & Cyclic (alkyl)(amino) Carbene \\
\hline OPV & Organic photovoltaic \\
\hline SF & Singlet Fission \\
\hline MRSF-TDDFT & Mixed Referance Spin-Flip Time-Dependent Density Functional \\
\hline \multicolumn{2}{|l|}{ Theory } \\
\hline SF-TDDFT & Self Consistence Time Dependent Density Functional Theory \\
\hline DFT & Density Functional Theory \\
\hline BHHLYP & Becke's half-and-half +Lee-Yang-Paar functional \\
\hline $\mathbf{Z P E}$ & Zero point Energy \\
\hline NEVPT2 & n-electron valence state perturbation theory 2 \\
\hline CAAC(F) & Fluorinated Cyclic (alkyl)(amino) Carbene \\
\hline CAAC(TFM) & Trifluoromethylated Cyclic (alkyl)(amino) Carbene \\
\hline NHC & N-heterocyclic Carbene \\
\hline HOMO & Highest Occupied Molecular Orbital \\
\hline LUMO & Lowest Unoccupied Molecular Orbital \\
\hline XRD & X-Ray Diffraction \\
\hline Dipp & Diisopropylphenyls \\
\hline LUNO & Lowest Unoccupied Natural Orbital \\
\hline SOMO & Singly Occupied Molecular Orbital \\
\hline $\mathbf{P h}$ & para-phenylene \\
\hline
\end{tabular}




\section{CHAPTER 1 INTRODUCTION}

\section{Section 1.1 Singlet Fission, an Optoelectronics Phenomenon}

Singlet fission is an interesting optoelectronics phenomenon that can enhance photoelectric conversion efficiency in organic photovoltaic devices. In a conventional organic photovoltaic device, the absorption of a solar photon in the chromophore layer generates a singlet exciton. We label this excitonic state as $S_{l}$ for its character of the lowest (1) singlet (S) excited state of the chromophore molecule. Such an exciton can hop to the chromophore-acceptor interface, where the exciton undergoes charge separation to a hole and an electron. The holes and electrons are accumulated in electrodes and become free charge carriers.
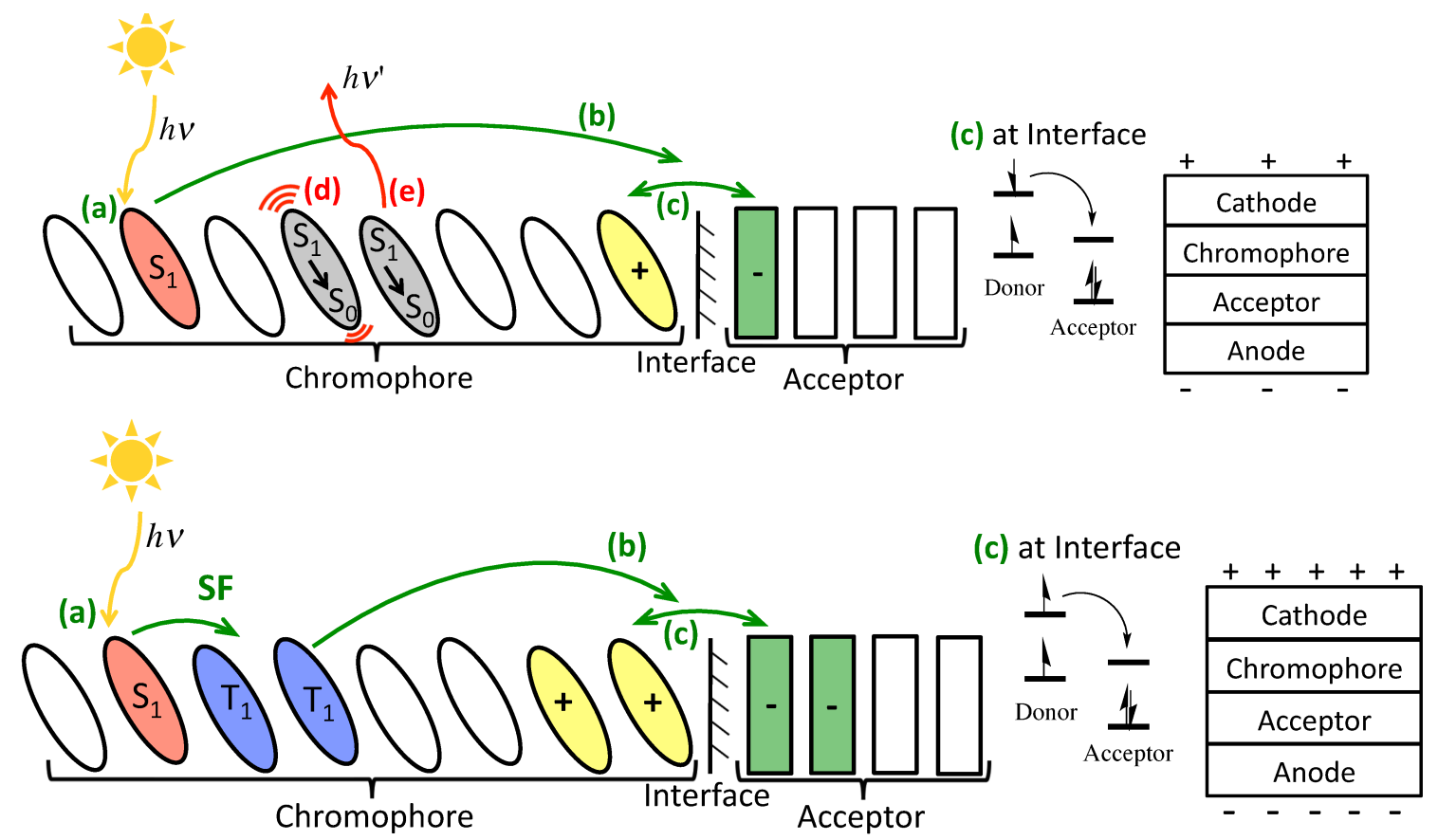

Figure 1. A comparison of photoelectric conversions without (upper panel) and with ( lower panel) singlet fission. There are common steps in both panels : (a) photoexcitation, (b) exciton migration, and (c) charge separation between chromophore and acceptor. Steps (d) and (e) are radiationless and radiative decays of singlet exciton. 
However, the $S_{1}$ exciton can undergo radiative and radiationless decays to the ground state (labeled as $S_{0}$ ), lowering the photoelectric conversion efficiency. All these steps are demonstrated in the upper panel, where the $S_{1}$ exciton is simplified to be localized in one chromophore molecule. In reality, the singlet exciton is delocalized and the range of delocalization is correlated with the bandwidth of the excitonic states, that is, singlet exciton transfer integrals.

Singlet fission (SF) is the process in which one $S_{l}$ exciton shares its energy with an adjacent chromophore that is in the ground state and converts to two triplet excitons on the two chromophores. ${ }^{1,2}$ The triplet excitons are labeled as $T_{1}$. SF starts with an internal conversion process, which typically occurs on a sub-ps to ps timescale ${ }^{3-7}$ and outcompetes the $S_{l}$ radiative and radiationless decays, which usually occur on ns timescales or longer. Molecules with ultrafast $S_{1^{-}}$to- $S_{0}$ radiationless decay, for example, azulene ${ }^{8}$ are not appropriate SF chromophores. The number of excitons is doubled in SF. Furthermore, owing to the spin selection rule, the $T_{1}$ excitons have a longer lifetime than $S_{l}$. The doubled number and longer lifetime enable more excitons to reach the interface and undergo charge separation, giving more electrons and holes at the electrodes. Therefore, SF provides an avenue to enhance photoelectric conversion efficiency. In their qualitative analysis, Hanna and Nozik showed that with SF, the conversion efficiency can surpass the approximately $1 / 3$ Schockley-Queisser limit ${ }^{9}$ of a single junction solar cell and reach approximately $1 / 2 .{ }^{10}$ Indeed, organic photovoltaic (OPV) devices that exhibit $>$ $100 \%$ quantum efficiency (electrons/photons ratio) have been fabricated, ${ }^{11-14}$ and the generation of more than one electron-hole pair by absorbing one photon is enabled by 
SF. A tandem architecture with a blue-absorbing SF donor and a red-absorbing acceptor is ideal for third generation solar cells. ${ }^{1,10,15,16}$

\section{Section 1.2 Electronic Structure Description of Singlet Fission}

A SF process is schematically depicted in Figure 2, in which a dimer model of chromophores A and B is used. As the triplet pair resides on two chromophores, dimers are the smallest systems that can have SF. They can be covalently connected dimers or two adjacent molecules in a van der Waals solid. In each chromophore, only the highest occupied and the lowest unoccupied molecular orbitals (HOMO and LUMO) are considered. A SF process consists of two steps, the spin-conserved step and the spindisentangled step. The spin-conserved step occurs within the singlet spin manifold and involves five electronic states: ${ }^{1,2}$ the single-excitonic states eg and ge, which are generated by photoexcitation; $t$, a singlet-coupled triplet pair state; and the chargetransfer (CT) states $c a$ and $a c$. The vertical arrows (electronic spins) and horizontal bars (orbital levels) in Figure 2 indicate occupation schemes and characteristic spin alignments of the states.

The second step shown in Figure 2 is the spin-disentangled step, in which the triplet pair loses its spin-coupling as an overall singlet and becomes two independent triplet states with random orientations. This step is induced by spin-dependent Hamiltonians, for example, the dipole-dipole interaction between electronic spins and the electron-nuclear spin interaction. ${ }^{1,17,18}$ These Hamiltonians do not commute with the total electronic spin operator. They mix the singlet-coupled triplet-pair state with the triplet- (if allowed by symmetry ${ }^{1}$ ) and quintet-coupled analogs. Along with thermalization, they 
randomize the orientations of the two triplets. These interactions are very weak, typically $<1 \mathrm{~cm}^{-1}$ in magnitude. This makes the disentanglement a slow process. A recent study that employed both transient absorption and time-resolved electron spin resonance spectroscopic techniques on 6,13-bis(triisopropylsilylethynyl)-pentacene (TIPSpentacene) covalent dimers showed that it took hundreds of nanoseconds to lose the spin correlation of the triplet pair. ${ }^{19}$ Similar experimental techniques were employed to study the 6,13-bis(triisobutylsilylethynyl)-pentacene dimer with a nonconjugated linker. ${ }^{20}$ It took hundreds of nanoseconds for the singlet-coupled triplet pair to convert to the quintet-coupled triplet pair, which is consistent with the findings in Reference 19. Furthermore, it took several microseconds to generate independent triplet excitons. The spin-disentanglement is so slow that the triplet states may have migrated apart, yet their spins are still correlated. Because of the small magnitude of the spin-dependent interactions, (pseudo)degeneracy of the singlet-, triplet-, and quintet-coupled triplet-pair states is necessary for the spin-disentanglement. When the two triplets reside on adjacent molecules, they may be ferromagnetically or antiferromagnetically coupled and the three spin manifolds are of different energies, hindering the disentanglement. When they dissociate to be nonadjacent, the (anti)ferromagnetic coupling is ineffective and the degeneracy is achieved. Therefore, dissociation facilitates the spin-disentanglement. 


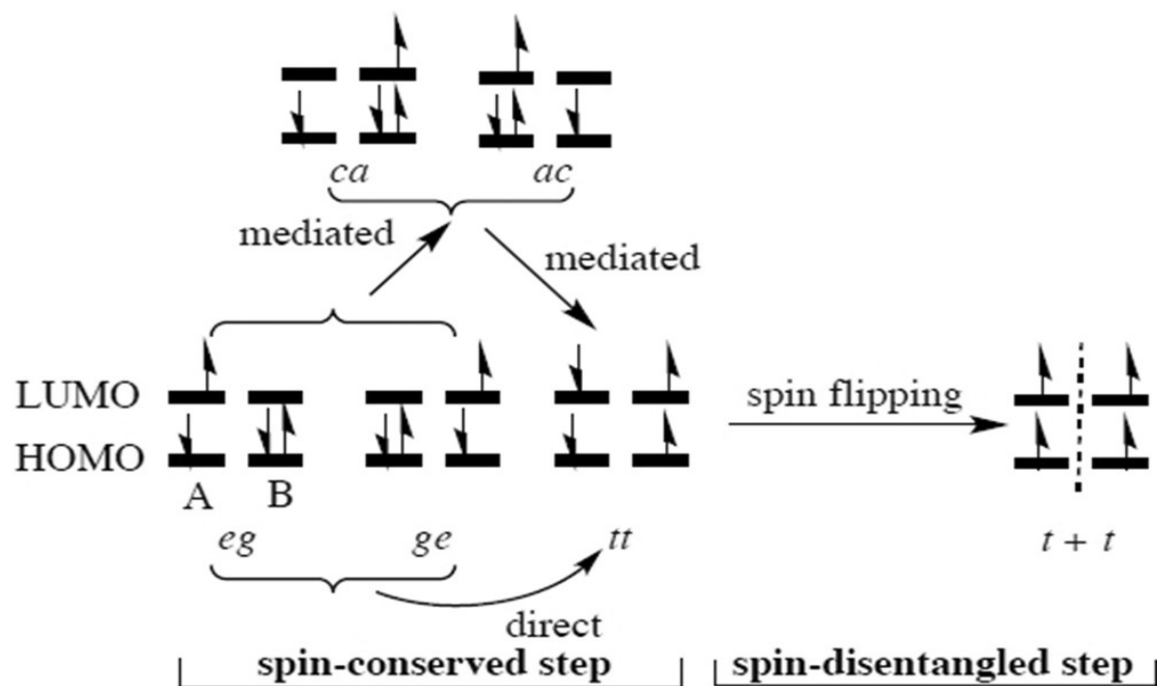

Figure 2. A schematic description of SF. The vertical arrows represent electronic spin. In the state labels, $e$ stands for the HOMO-to-LUMO singlet exciton, $t$ is the triplet exciton, $g$ is the ground state, $c$ is the cationic state, and $a$ is the anionic state. A vertical dashed line is added to the $t+t$ state to emphasize that the two triplets are independent of each other. This figure is taken from Ref. 80.

We need to emphasize that Figure 2 gives a simplified description of SF. It is most relevant for SF across a dimer. It cannot describe the delocalization of the singleexcitonic state beyond two monomers, neither the migration of the triplets. Also, the CT states may have different energies when the two chromophores are immersed in a solid environment. Another important difference is in the number of states (or density of states). In a dimer, there are two single-excitonic states and one multi-excitonic state. As the size of the oligomer grows, the number of single-excitonic states grows linearly, whereas that of multi-excitonic states grows quadratically. In combination with triplet migration, this faster growth of multi-excitonic states provides an entropy driving force for SF. 


\section{Section 1.3 The Role of Vibronic Interactions}

SF is an energy transfer process. Like in other energy transfer processes, vibronic coupling plays a significant role in SF. The five diabats and their analogs in more extended systems can be viewed as forming the electronic system, which is coupled to a bath of vibrational degrees of freedom (phonons in solids). ${ }^{21}$ Thermodynamically speaking, the bath dissipates the energy released in the SE-to-ME conversion if it is an

exoergic process. For an endoergic SF process, which can still be driven by entropy, ${ }^{7,22}$ the bath provides the needed energy.

The couplings of the diagonal matrix elements are called the Holstein couplings, and those of the off-diagonal elements are called the Peierls couplings. ${ }^{23}$ The Holstein couplings bring about different potential energy surfaces (PESs) for different diabats. Figure 3 (a) demonstrates the effects of Holstein couplings in an ideal SF process. The Franck-Condon excitation $(h v)$ vertically shifts the ground-state (GS) vibrational wave function to the SE potential energy surface (simplified as a curve in the figure, with an abstract vibrational coordinate q). The ground-state structure corresponds to an interaction region where the $\mathrm{SE}$ and $\mathrm{ME}$ energies are close and the SE-to-ME transition induced by the matrix element $H_{S E, M E}$ is efficient, resulting in a vibrational wave packet on the ME PES. $H_{S E, M E}$ is assumed to be fixed in this model. The back-and-forth population conversion between $\mathrm{SE}$ and $\mathrm{ME}$, which is the Rabi oscillation, continues. Meanwhile, the wave packets on the different PESs evolve following the respective potentials and migrate away from the vertical interaction region. All wave packets in the figure are simplified as gaussians. Given that the ME PES has a minimum lower than the 
SE PES, the ME wave packet explores structures with larger SE-ME energy gaps than the SE wave packet. Consequently, the ME-to-SE conversion is slower than the SE-toME conversion. The efficiency of a $H_{S E, M E}$-driven conversion is reflected by the transparency of the relevant arrow in Figure 3 (a): the more transparent, the slower. These unbalanced conversion rates remove the Rabi oscillation and result in an overall unidirectional SE-to-ME conversion. Naturally, as the number of the coupling modes, that is, the dimension of the PESs, increases, the conversion is less reversible. Again, the argument here is based on the dimer model. The entropy effect that favors the formation of the ME state has not been considered yet.
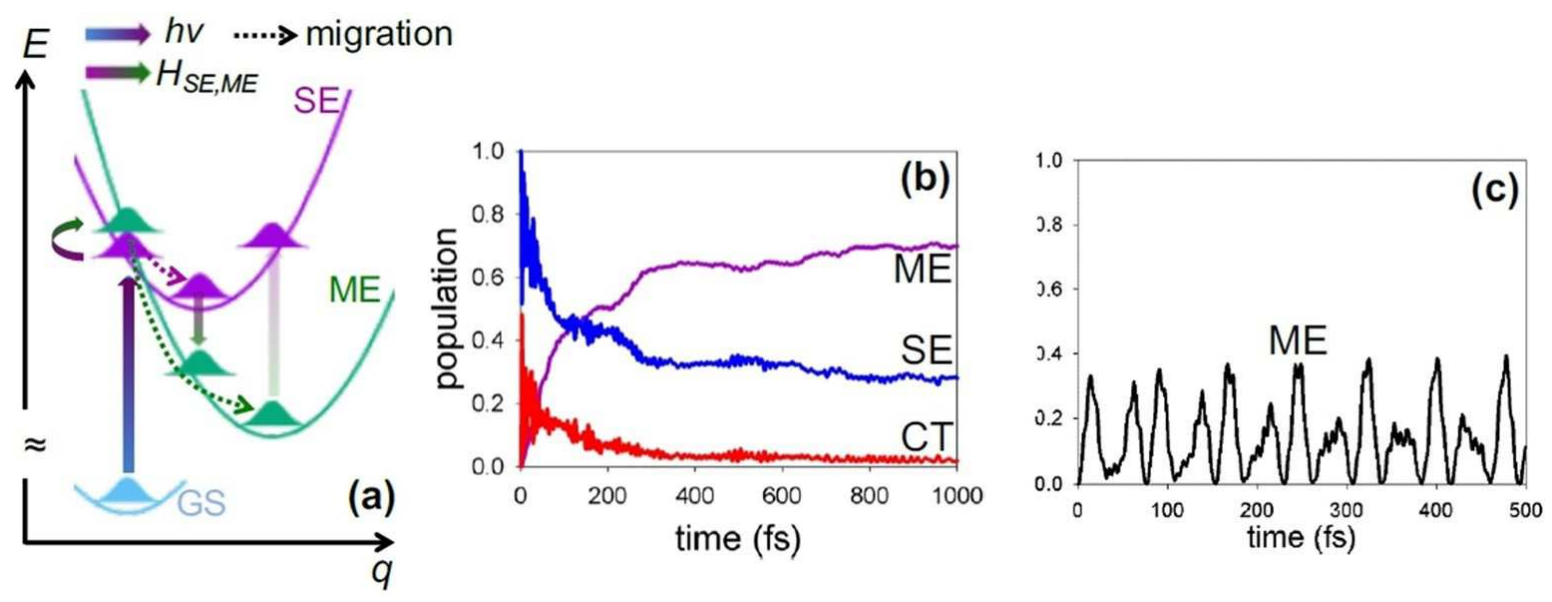

Figure 3. (a) Vibrational wave packets on different potential energy surfaces and their transitions to different states in SF; evolutions of diabatic populations in a SF process from dynamics simulations that consider (b) vibronic interaction and (c) only the electronic Hamiltonian. In panel (c), only the ME state population is plotted to show the Rabi oscillation. Panels (b) and (c) are adapted from Ref. 24.

The significant distance between minima in PESs leads to a small overlap between the SE and ME wave packets, that is, a decoherence between the two states. The decoherence provides another way to understand why vibronic coupling destroys the 
Rabi oscillation. ${ }^{24}$ If the Franck-Condon region is far from the interaction region, the Holstein coupling may bring the SE wave packet to the interaction region. Typical evolutions of populations in SF from dynamics simulations with and without considering vibronic interactions are compared in Figure 3 (panel (b) vs. panel (c)). As elucidated above, the former exhibits a unidirectional SE-to-ME population conversion whereas the latter shows a Rabi oscillation. Evidently, there is no SF if there are no vibronic couplings.

\section{Section 1.4 Searching for Singlet Fission Chromophores}

SF was first observed in crystalline anthracene (1) in $1965^{25}$ and its studies were immediately extended to tetracene (2) and pentacene (3). ${ }^{26-31}$ A few materials are known to undergo SF efficiently. They include tetracene, pentacene, some of their derivatives, ${ }^{4,6,7,11,12,32-43}$ perylene diimide, ${ }^{44}$ as well as several conjugated polymers. ${ }^{45-50}$ An emerging class of SF chromophores are non-polycyclic thienoquinoid compounds. ${ }^{51}$ This highly limited arsenal of SF chromophores obstructs the application of SF in OPVs. This difficulty motivated a series of studies in understanding the intrinsic characters of the chromophores and searching for new chromophores.

The key energetic requirements for SF chromophores are,

$$
\begin{aligned}
& E\left(S_{1}\right) \geq 2 E\left(T_{1}\right) \\
& E\left(T_{2}\right) \geq 2 E\left(T_{1}\right) \\
& E\left(Q_{1}\right) \geq 2 E\left(T_{1}\right)
\end{aligned}
$$

$S, T$ and $\mathrm{Q}$ are used to label states in singlet, triplet and quintet spin manifolds. The subscripts 1 and 2 indicate the first and second excited states in the respective spin manifolds. The first inequality guarantees that the fission of one $S_{1}$ exciton to two $T_{1}$ excitons is exoergic and thermodynamically favorable. The second 
inequality ensures that the fusion of two SF generated $T_{1}$ excitons to one $T_{2}$ exciton is endoergic and thermodynamically unfavorable. In terms of spin angular momentum addition, two $T_{1}$ excitons can also fusion to one $Q_{1}$ exciton (the lowest quintet excited state). However, $E\left(Q_{1}\right)$ is usually greater than $2 E\left(T_{1}\right)$ and the fusion is hindered as shown in the last inequality.
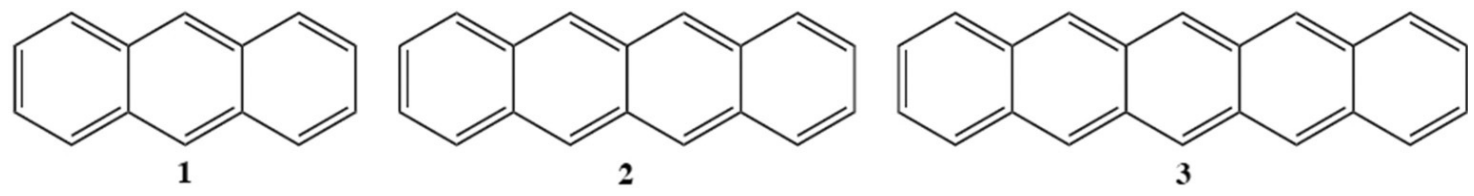

$T_{1}$ typically involves exciting an electron from HOMO to LUMO and flipping its spin, whereas $Q_{l}$ involves the second excitation and spin-flipping from HOMO-1 to LUMO+1, which costs more energy than the first excitation. Therefore, Equation (3) is usually satisfied and can be ignored. Two $T_{1}$ excitons are unlikely to recombine to one molecule in $T_{l}$ and one molecule in $S_{0}$ owing to the substantial energy release by $E\left(T_{l}\right)$ (i.e., the energy gap law). Instead, they may recombine to form one molecule in $T_{2}$ and the other in $S_{0} ; E\left(T_{2}\right)$ might be close to $2 E\left(T_{1}\right)$. Equations (1) and (2) guide the search for SF-capable chromophores. As $S_{1}$ usually involves HOMO-to-LUMO excitation (Class I chromophores defined in Ref. [1]) whereas $T_{2}$ involves excitation with a larger orbital energy gap, for example, HOMO-to-LUMO+1, HOMO-1-to-LUMO, etc., $E\left(T_{2}\right)$ is likely to be larger than $E\left(S_{I}\right)$. Therefore, we can by and large focus on Equation (1). However, $E\left(T_{2}\right)$ should always be examined in designing SF chromophores. In addition to Equation (2), $E\left(T_{2}\right)>E\left(S_{1}\right)$ is desired. ${ }^{1,2}$ This inequality ensures a thermodynamically unfavored $S_{l}$-to- $T_{2}$ intersystem crossing (ISC), which is followed 
by the efficient $T_{2}$-to- $T_{1}$ decay (Kasha's rule ${ }^{52}$ ); only one $T_{1}$ exciton results. Although $E\left(T_{2}\right)>E\left(S_{1}\right)$ is desired, it is not as a strong condition as Equations (1) and (2). The states involve p- to-p excitations. According to El-Sayed's rule, ${ }^{53,54}$ ISC between $\pi$ excited states is usually slow, although it may be thermodynamically favorable. The energies in Equations (1) to (3) should be those of the optimized structures of the respective states, because vibrational relaxation is competitively fast compared with the fission and fusion. Other than the energy relations, $E\left(T_{l}\right)$ close to $1 \mathrm{eV}$ is desired. This gap maximizes the efficiency in energy conversion. ${ }^{1}$

One usually does not need to consider states higher-lying than those in Eqs. 1-3. According to the Kasha's rule, those higher-lying states $\left(S_{3}, T_{3}, Q_{2}\right.$, etc.) are usually short-lived. They decay to the lower-lying excited states rapidly through non-adiabatic vibronic interaction.

\section{Section 1.5 Alternant Hydrocarbons and Diradicals; The Two Limits}

The structural search has been concentrated on $\pi$-conjugated systems because of their good absorption in an extensive range of photon frequencies. The first systematic search for chromophores that satisfy Equation (1) was performed by Paci et al. ${ }^{55}$ They proposed two classes of parental structures that can be modified to meet the requirement: the closed-shell alternant hydrocarbons in the left side of Figure 4 and the open-shell diradicals in the right side. When both $S_{1}$ and $T_{1}$ involve one-electron HOMO-to-LUMO excitation, their energies differ by twice the exchange integral between the two orbitals $\left(2 K_{h l}\right)$. For alternant hydrocarbons, this gap is largely invariant. ${ }^{1}$ Therefore, by reducing $E\left(S_{I}\right)$ (or likewise the HOMO-LUMO gap), we will 
eventually reach the structures that satisfy $E\left(S_{1}\right) \geq 2 E\left(T_{1}\right)$. Indeed, as the length of acene increases and the HOMO-LUMO gap decreases,${ }^{56}$ all acenes longer than and including tetracene satisfy this condition. At the other end, diradicals have triplet ground states, whereas the lowest singlet $\left(S_{0}\right)$ is higher by twice the exchange integral between the two singly occupied orbitals A and $\mathrm{B}\left(2 K_{\mathrm{AB}}\right) . E\left(S_{l}\right) \geq 2 E\left(T_{1}\right)$ is automatically satisfied. However, diradicals are in general unstable. By increasing the overlap between orbitals $\mathrm{A}$ and $\mathrm{B}$, and hence the HOMO-LUMO gap (HOMO:the bonding orbital between $\mathrm{A}$ and B; LUMO : antibonding), the resultant more stable diradicaloid has $S_{0}$ as the ground state and $E\left(T_{1}\right)$ increases. When $E\left(T_{1}\right)$ increases to near but still less than $\frac{1}{2} E\left(S_{1}\right)$, a promising SF chromophore is reached. The two classes of structures are not mutually exclusive, as the HOMO-LUMO gap and diradical character are correlated. ${ }^{56-58}$

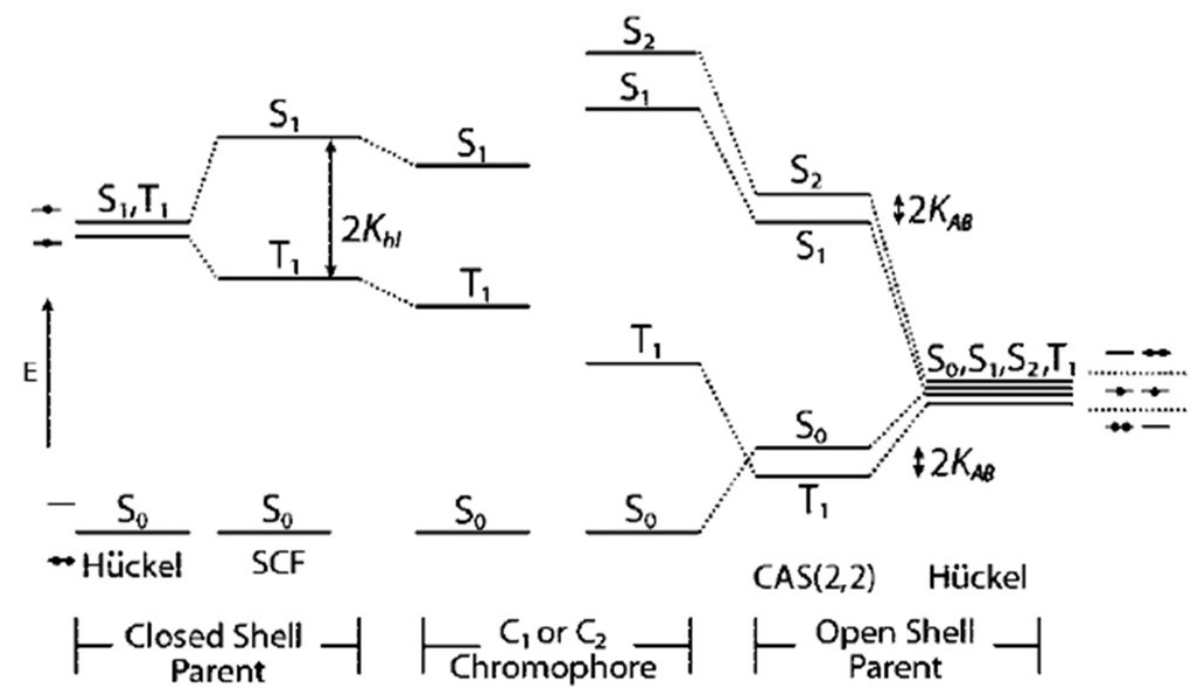

Figure 4. Characteristic energy levels of the closed- and open-shell parental structures (at the left and the right end), and their changes when the structures are perturbed towards singlet fission chromophores (in the middle). This figure is taken from Ref. 1. 
Paci et al. used the efficient Pariser-Parr-Pople (PPP) method ${ }^{59,60}$ to calculate geometrically relaxed $E\left(S_{1}\right), E\left(T_{1}\right)$, and $E\left(T_{2}\right)$ values for more than 60 structures of the two classes. Several $o$ - and $p$-xylylene derivatives were predicted to be promising chromophores. Among them, 1,3-diphenylisobenzofuran (4, DPB) has been shown to undergo SF. ${ }^{61,62,63}$ DPB is the first theoretically designed chromophore that turned out to undergo SF. It has attracted lots of research interest. ${ }^{61-65}$

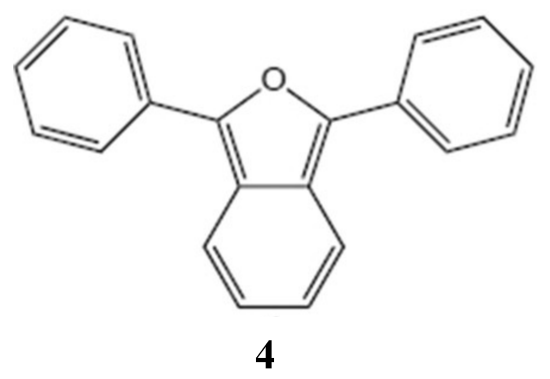

\section{Section 1.6 A Unified Diradical Character View of the SF Chromophores}

Considering the close connection between the two classes of parental structures for SF chromophores, Minami and Nakano developed a unified view for searching for SF chromophores based on multiradical character. ${ }^{66}$ They used a simple linear $\mathrm{H}_{4}$ model of $D_{\infty h}$ symmetry to investigate the relationship between excitation energies and diradical/tetraradical character. Such a model with four orbitals and four electrons $(4 \mathrm{o} 4 \mathrm{e})$ allows to simulate the $T_{2}$ state of a chromophore. By varying the three $\mathrm{H}-\mathrm{H}$ internuclear distances, they freely tuned the ground-state character of the $\mathrm{H}_{4}$ model from a perfect closed- shell $(\mathrm{H}-\mathrm{H} \cdots \mathrm{H}-\mathrm{H})$, to a diradical $(\mathrm{H} \cdots \mathrm{H}-\mathrm{H} \cdots \mathrm{H})$, and to a tetraradical $(\mathrm{H} \cdots \mathrm{H} \cdots \mathrm{H} \cdots \mathrm{H}) . \quad E\left(S_{1}\right)-2 E\left(T_{1}\right)$ and $E\left(T_{2}\right)-2 E\left(T_{1}\right)$ were calculated for each $\mathrm{H}_{4}$ conformation and plotted against the occupation numbers $\left(y_{0}\right.$ and $\left.y_{1}\right)$ of the lowest unoccupied natural orbital (LUNO) and the second lowest one (LUNO+1), which, 
respectively, indicate the diradical and tetraradical characters of a molecule. Through inspecting the variations of the two differential energies with respect to $y_{0}$ and $y_{1}$, the authors proposed the following guidelines for the chromophore search: 1) the chromophore should have considerable but not too much diradical character in order to satisfy $E\left(S_{1}\right)-2 E\left(T_{1}\right)$ greater than and close to 0 , so that the energy efficiency in SF is high; 2) the tetraradical character of a chromophore should be minute to satisfy $E\left(T_{2}\right)$ $2 E\left(T_{1}\right)>0$.

The diradical character can be enhanced by increasing the aromaticity of a fragment of a molecule. Ito and Nakano investigated a series of heteroacene models based on core structures of $m$-xylylene $(\mathrm{N} m-i, \mathbf{5}) \cdot{ }^{67}$ The authors tuned the aromaticity of the central rings by $\mathrm{N}$-substitution (changing the $m$ value) and modified the $\pi$ conjugation extension (changing the $i$ value). The increases of the aromaticity and conjugation length of the central rings strengthen the diradical character with the resonance structure shown in $\mathbf{5}$, and the model molecules start to satisfy Equation (1). N2-4 and N0-3 were identified as ideal SF chromophores: they satisfy the energy criteria and have $E\left(T_{1}\right)$ close to $1 \mathrm{eV}$.

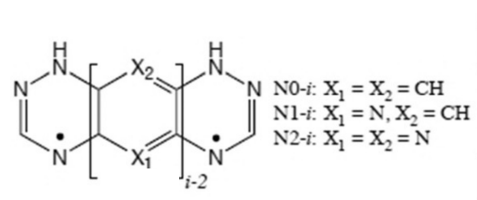

5

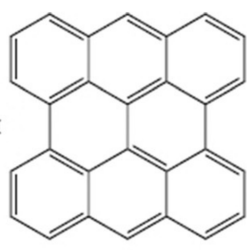

6

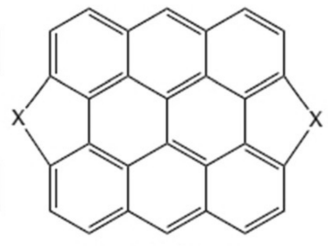

6' $^{\prime} \mathrm{X} \mathrm{X}=\mathrm{C}_{2} \mathrm{H}_{2}, \mathrm{O}, \mathrm{S}$

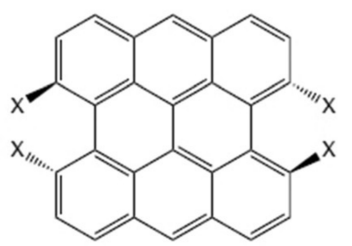

6"- X X=F, Cl

One way to design SF chromophores is to start with a structure with too much diradical character and with $2 E\left(T_{1}\right)<E\left(S_{I}\right)$, and then introduce substitutions to mitigate 
the diradical character to make $2 E\left(T_{1}\right)$ closer to $E\left(S_{l}\right)$. Following this route, Ito et al. designed SF chromophores with bisanthene (6) as a starting structure. ${ }^{6}$ Bisanthene possesses too significant diradical character. Its calculated $E\left(S_{l}\right)$ and $E\left(T_{l}\right)$ values were 2.393 and $0.939 \mathrm{eV} .2 E\left(T_{l}\right)-E\left(S_{l}\right)=-0.515$ indicates a substantial energy loss in SF. Ito et al. reduced the diradical character by making $\mathrm{O}, \mathrm{S}$, and $\mathrm{C}_{2} \mathrm{H}_{2}$ substitutions as in 6'-X. The substitutions introduce two extra sextet rings to the non-diradical resonance structure and hence reduce the diradical character. The authors also substituted bisanthene with bulky halogen atoms and methyl groups as in 9"-X to twist the structure to non-planar. The non-planarity reduces the overlap between the frontier orbitals of the two anthracene units and enlarges the HOMO-LUMO gap, reducing the diradical character. Compounds 6'-O and 6"-F were predicted to be promising chromophores, with $2 E\left(T_{1}\right)-E\left(S_{1}\right) \approx 0$.

\section{Section 1.7 Small SF Chromophores}

Conventional SF chromophores such as tetracene, pentacene, and their derivatives are fairly large in size. Akdag et al. pioneered the design of small SF chromophores. ${ }^{69}$ Small chromophores are desired because: 1) they can give high exciton density, which eventually facilitates the development of mini OPV devices ; 2) although they may not be stable, they serve as core structures for more kinetically persistent derivatives; 3) they are convenient models for theoreticians to investigate $\mathrm{SF}$, like $\mathrm{CH}_{2}$ for carbene chemistry research. The authors proposed five monocyclic structures. They are five- or sixmembered aromatic rings with endocyclic electron donors $\left(s p^{2} \mathrm{~N}\right)$ and acceptors (carbonyl). Compound 7 is shown as a representative. The donors and acceptors were introduced to exert the captodative effect ${ }^{70}$ to stabilize radical resonance structures for the 
moieties that they sandwich. With a pair of such sandwiched moieties, the diradical character of 7 is enhanced. The authors calculated low-lying excitation energies for the five designed structures by using the second-order Complete Active Space Perturbation Theory (CASPT2) method with active spaces that span all $\pi$ and lone pair orbitals. Such large active spaces can only be used for small molecules. Only 7 was found to satisfy Equation (2) and almost satisfy Equation (1) (close to isoergic SF), as well as $E\left(S_{1}\right)<$ $E\left(T_{2}\right)$. Its small size should be emphasized, especially when compared with tetracene (2) and pentacene (3).

The success of the captodative strategy motivated Zeng et al. to follow the same route, but employ a special acceptor, the endocyclic $\mathrm{s} p^{2} \mathrm{~B}$ atom. ${ }^{71}$ The authors proposed a series of azaborine(BN)-substituted mono- and bi-cyclic aromatic structures. They calculated excitation energies of the structures by using the General MultiConfigurational Quasi-Degenerate Perturbation Theory (GMC-QDPT). Three structures (8 to 10) were found to be promising chromophores, and the smallest $\mathbf{8}$ is even smaller than 7. Disregarding the methyl groups on $\mathrm{N}$ in 7 and recognizing the similarity between $\mathrm{BF}$ and $\mathrm{CO}, \mathbf{7}$ and $\mathbf{8}$ are isoelectronic and isosteric. Through this work, the authors attempted to crosslink the two vibrant fields of singlet fission and azaborine chemistry. ${ }^{72}$

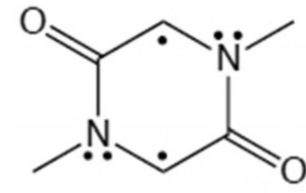

7<smiles>FB1CNB(F)CN1</smiles>

8

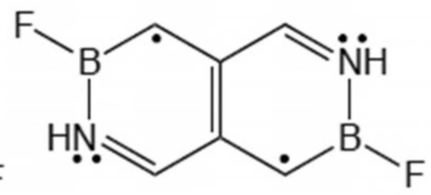

9

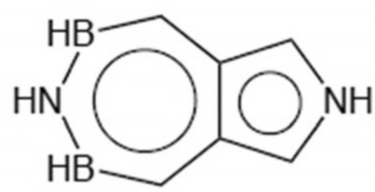

10 
In Refs. [69] and [71], the two captodatively stabilized radical centers are not in contact. For instance, $\mathbf{8}$ can be viewed as being composed of two BN-substituted methyl groups, in which the $\mathrm{C}$ sites are not connected. Wen et al. approached the problem from another direction. ${ }^{73}$ They started with two radical centers in contact, that is, they are covalently coupled, and then reduced the coupling and increased their diradical character by introducing donors and acceptors. The reduction in interaction is illustrated in Figure $\mathbf{8}$, where we compare the $\pi$-bonding orbitals of the plain (upper) and a captodatively modified (lower) ethylene. The acceptor (donor) delocalizes the $\pi$ singly occupied orbital of methylene through having a bonding (antibonding) interaction with the central C. The resultant smaller amplitudes at the central $\mathrm{C}$ atoms reduce the $\pi-\pi$ overlap, and hence a smaller HOMO-LUMO gap is obtained.
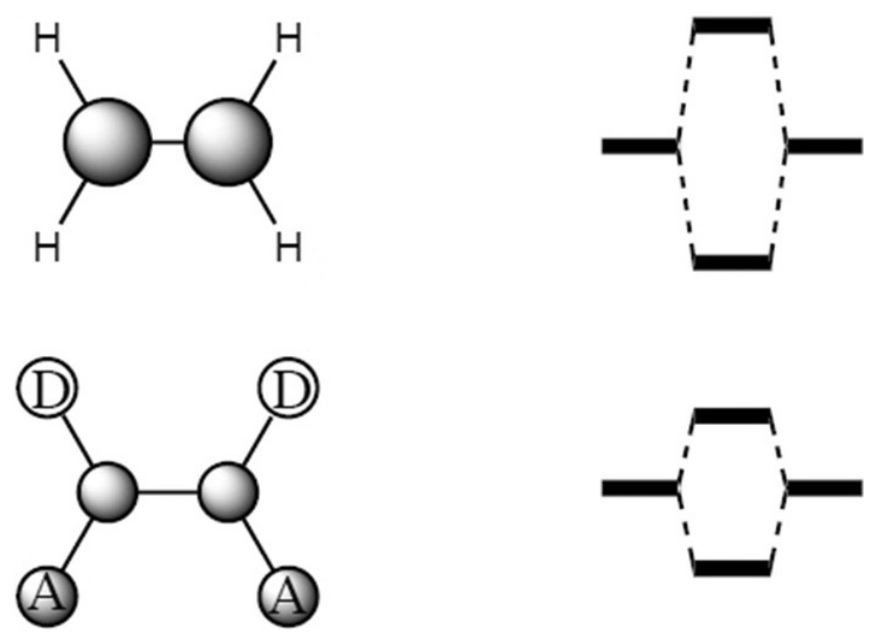

Figure 5. Weakening of the covalent interaction between two methylene radicals in forming the $\pi$ bond of ethylene when they contain acceptor (A) and donor (D) substituents. 
Through density functional theory (DFT) and time-dependent density functional theory (TDDFT) calculations, Bhattacharyya et al. designed a small SF chromophore based on mono-Si-substitution on anthracene $(\mathbf{1 1}){ }^{74} \mathrm{Si}$ is an electron donor and its endocyclic substitution raises the HOMO energy more than the LUMO energy. The reduced HOMO-LUMO gap increases the diradical character of $\mathbf{1 1}$ and makes it satisfy Equation (1), with $E\left(S_{1}\right)=2.65$ and $E\left(T_{1}\right)=0.97 \mathrm{eV}$. The authors further introduced a CN group to $\mathbf{1 1}$ and the resultant structure (12) was shown to have almost isoergic SF, with $E\left(S_{1}\right)-2 E\left(T_{1}\right)=0.05 \mathrm{eV}$.

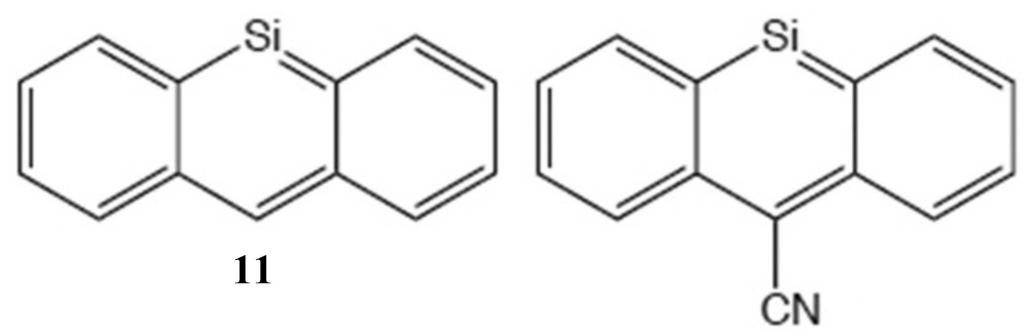

12 


\section{Section 1.8 Summary of Promising Theoretical Design}

Table 1. Excitation energies (in $\mathrm{eV}$ ) of theoretically designed promising $\mathrm{SF}$ chromophores.

\begin{tabular}{c|ccc}
\hline \multicolumn{1}{c}{ STRUCTURE } & $\boldsymbol{E}\left(\boldsymbol{S}_{\boldsymbol{0}}\right)$ & $\boldsymbol{E}\left(\boldsymbol{T}_{\boldsymbol{l}}\right)$ & $\boldsymbol{E}\left(\boldsymbol{T}_{\boldsymbol{2}}\right)$ \\
\hline $\mathbf{4}$ & 3.01 & 1.41 & 3.16 \\
$\mathbf{5} \mathbf{~ N 2 - 4}$ & 2.60 & 1.08 & 2.75 \\
$\mathbf{5} \mathbf{N 0 - 3}$ & 2.41 & 1.10 & 2.51 \\
$\mathbf{6}$ '- $\mathbf{O}$ & 2.80 & 1.36 & 2.92 \\
$\mathbf{6}$ "- F & 2.52 & 1.26 & 2.72 \\
$\mathbf{7}$ & 2.11 & 1.14 & 3.52 \\
$\mathbf{8}$ & 3.01 & 1.34 & 5.26 \\
$\mathbf{9}$ & 1.99 & 0.97 & 2.82 \\
$\mathbf{1 0}$ & 2.03 & 0.84 & 3.00 \\
$\mathbf{1 1}$ & 2.65 & 0.87 & - \\
$\mathbf{1 2}$ & 2.57 & 1.26 & - \\
\hline
\end{tabular}

The calculated excitation energies of the promising SF chromophores discussed above are summarized in Table 1 for comparison. Among them, 7, 9, and $\mathbf{1 2}$ are of special interest. Their $E\left(T_{l}\right)$ values are close to the optimal $1 \mathrm{eV}$ gap and $E\left(S_{l}\right)$ values are close to twice the $E\left(T_{I}\right)$ values, minimizing energy loss in SF. Also, the close to $2 \mathrm{eV} S_{0}$-to- $S_{I}$ absorption occurs at the frequency range with fairly high spectral irradiance in the solar spectrum. ${ }^{75}$ They are high value targets for future syntheses and experimental investigations. 


\section{Section 1.9 Design of SF Chromophores with Cyclic (Alkyl)(Amino) Carbene Building Blocks, the Motivation and Objective of This Work}

As introduced in the previous subsections, one obstacle for the application of the SF phenomenon in photovoltaics is that only a small number of molecules meet the three criteria of Equations (1)-(3). Among them Equations (1) and (2) are more difficult to meet. Especially, current research of SF is "overwhelmingly limited" to tetracene ${ }^{7}$ and pentacene. ${ }^{6,12,76}$ There is thus a strong motivation to design more SF chromophores that meet the criteria. It has been clarified that the possession of non-negligible diradical character and the lack of tetraradical character are the underlying reasons for a molecule to satisfy the two inequalities, respectively. ${ }^{55,66,77,78,79}$ Stable molecules, either isolated in reality or optimized in silico, rarely possess significant tetraradical character. Therefore, it would be relatively easier to satisfy Eq. 2 than Eq. 1, which is thus the primary criterion for SF chromophores.

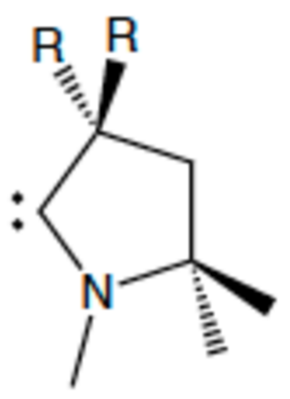

13

We have also learned that the enhancement of diradical character can be realized by introducing captodative effect. ${ }^{69,73,76,80-82}$ These modifications shall be applied to structures that are by themselves not too low in diradical character. Otherwise, the 
enhancement in diradical character may not be enough to satisfy Eq.1. Recently, attentions have been drawn to structures with cyclic (alkyl)(amino)carbenes (CAACs) as building blocks shown in $\mathbf{1 3}{ }^{83}$ CAACs are persistent singlet carbenes ${ }^{84}$ Last year, a modular approach for connecting CAACs by spacers to form stable Kekulé diradicaloids was reported by the Bertrand group. ${ }^{85}$ This approach allows tuning the diradical character of the CAAC-based diradicaloids through modifying the spacer, especially its length and aromaticity. Therefore, this approach opens the doorway to design CAAC-based SF chromophores. Immediately following this work, Messelberger et al. performed a computational study of a series of CAAC-spacer-CAAC dimer structures and identified several candidates as SF chromophores. ${ }^{83}$ It is noteworthy that while CAACs are usually used as stabilizers for radical and diradical moieties attached to them ${ }^{86-90}$ they themselves are radical centers in those SF chromophore candidates.

Inspired by these latest advances in the field of SF, we in this work perform quantum chemistry calculations to study the possibility of designing CAAC-spacerCAAC SF chromophores through fluorination and trifluoromethylation at the $\alpha \mathrm{C}$. The ideas of fluorination and trifluoromethylation originate from the captodative effect: by stabilizing a radical center by simultaneously attaching a donor and an acceptor to it. ${ }^{91}$ By connecting a CAAC unit to a spacer, one electron of the carbene's $\sigma$ lone pair participates in forming a $\sigma$ bond with the spacer. The leftover unpaired electron occupies the $\pi$ orbital of the carbene, making the carbene a $\pi$ radical. The $\mathrm{N}$ atom adjacent to the carbene $\mathrm{C}$ exerts a donating effect to the singly occupied carbene $\pi$ orbital. If the $\alpha \mathrm{C}$ can serve as a $\pi$ acceptor, or at least as a poorer $\pi$ donor, the CAAC $\pi$ radical unit will be stabilized by the captodative effect of the $\alpha \mathrm{C}$ and the $\mathrm{N}$, and the diradical character of the CAAC- 
spacer-CAAC structure will be enhanced. The structure is then more likely to satisfy Eq. 1. According to the work shown below, fluorination and trifluoromethylation at the $\alpha \mathrm{C}$ does enhance its $\pi$ accepting capability. Only homodimers are considered in this work, since heterodimers are likely to feature more or less ionic character in their ground state, which is exclusive with diradical character.

\section{Section 1.10 Organization of the Rest of the Thesis}

In Chapter 2, details of the theoretical methods used in our study are given. In Chapter 3, the computational results are presented and discussed. The conclusions are given in Chapter 4. The introduction in Chapter 1 is included in the review article published as A. Japahuge and T. Zeng "Theoretical studies of singlet fission: searching for materials and exploring mechanisms (review)." ChemPlusChem 2018, 83, 146-182. The review article was highlighted as a Very Important Paper of the journal. The material designing study in this thesis has been published as A. Japahuge, S. Lee, C. H. Choi, and T. Zeng, "Design of Singlet Fission Chromophores with Cyclic (Alkyl)(Amino) Carbene Building Blocks." J. Chem. Phys. 2019, 150, 234306. 


\section{CHAPTER 2 METHODOLOGY}

\section{Section 2.1 MRSF-TDDFT Method for Electronic Structure Correlation}

Schrödinger equation is the fundamental equation in quantum mechanics. There are two forms of the equation, the time-independent and the time-dependent Schrödinger equation. We need to solve the time-independent Schrödinger equation for the designed chromophores to obtain their excitation energies. However, it is difficult to solve Schrödinger equations for many-body systems. In practice, we rely on approximations to solve the equations. And the accuracies of the solutions are critically dependent on the approximations and whether they are applicable for the systems of interest.

Density functional theory (DFT) delivers a class of efficient methods to solve Schrödinger equations for multi-electronic systems. Traditional DFT methods are not reliable for systems whose wave functions are of multi-reference nature, i.e., that wave functions that are not dominated by one Slater determinant (or electron configuration). SF chromophores fall into this type of systems. The significant diradical character in their ground state wave functions determines the multi-reference nature of their ground state wave functions. Their excited states, with excitations out of the ground states, are also characterized by substantial contributions by several Slater determinants. In general, it is desired to develop DFT methods to handle systems with multi-reference wave functions accurately and efficiently. Such methods are especially needed for our study of designing SF chromophores.

Recently, two of our collaborators, Seunghoon Lee and Cheol Ho ChoI, developed the mixed-reference spin-flip time-dependent density functional theory (MRSF-TDDFT) formalism ${ }^{92}$ and its analytic energy gradient. The new theory is an 
improved SF-TDDFT, which can treat both dynamic and non-dynamic correlations in a balanced way. This means it can be used to study systems with multi-reference wave functions. This theory overcomes the notorious deficiencies of the conventional SFTDDFT known as the spin-contamination problem. It is shown that MRSF-TDDFT gives improved results than those of SF-TDDFT in calculating excitation energies. ${ }^{93}$ In the MRSF-TDDFT, the lowest triplet state obtained at the restricted open-shell DFT level is used as the reference state. The singlet and triplet response states of the same molecular structure are obtained by the linear response, i.e., one-electron transitions, from the same reference state, which is similar to configuration interaction singles.

Although the molecular orbitals of the reference states are optimized for the lowest-triplet state, the singlet and triplet response states are described by rotations from occupied orbitals to virtual orbitals of reference state. Therefore, the MRSF-TDDFT method does not bias towards the triplet response state that resembles the reference state. Geometry optimizations for all studied structures, in their ground and excited states in both singlet and triplet spin manifolds, are performed using the MRSF-TDDFT method. The method is also used for all hessian calculations.

\section{Section 2.2 Geometry Optimization}

The process of finding a settlement of a molecule where the intra-atomic forces among atoms are relatively closer to zero is called as energy minimization or geometry optimization. It is equivalent to locating stationary points on potential energy surface. As to our chromophore designs, all reported optimized dimer structures are of $C_{2}$ symmetry, unless further specified. Optimizations with $C_{i}$ symmetry for several representative molecules give similar energies and structural parameters. For instance, the (CAAC) ${ }_{2}-\mathrm{C}_{2}$ 
dimer structures with $C_{2}$ and $C_{i}$ symmetries have identical bond lengths at their cumulene fragments. Their calculated vertical $E\left(S_{1}\right) \mathrm{s}$ and $E\left(S_{2}\right) \mathrm{s}$ differ by only 0.002 and $0.006 \mathrm{eV}$, respectively. Hence, the discussion will be focus only on the $\mathrm{C}_{2}$ structures. The BHHLYP functional ${ }^{94,95}$ and the Def2-SVP basis set $^{96}$ are used in the optimization and hessian calculations, which are performed using a development version of the GAMESS-US program package..$^{97,98}$ The hessian calculations confirm that the optimized structures are true minima on potential energy surfaces. Optimization of the water dimer structure is done by the Def2-SVP and Def2-TZV PP basis sets and the BHHLYP functional. The resultant O-O distances are 2.86 and $2.90 \AA$, respectively. The $0.04 \AA$ small difference corroborates the competence of the economical Def2-SVP basis set in optimizing structures with hydrogen bonding interaction. Hydrogen bonding interaction plays an important role in determining the structures of the dimers that are considered in this study. All the reported energies contain Zero Point Energy (ZPE) corrections, unless further specified. The rapid geometry relaxation associated with photoexcitation competes with SF. ${ }^{1}$ It is hence more reasonable to use ZPE-corrected minimum-tominimum (i.e., adiabatic) excitation energies to judge whether a molecule satisfies Eqs. 1 and 2. As shown below, structural reorganizations induced by excitation can be significant. It is well known that such excitation-induced reorganizations play an important role in energy transfer and charge transfer of organic semiconductors. ${ }^{99-101}$ In general, significant reorganizations lead to higher barriers and lower rates of the transfers.

\section{Section 2.3 Excitation Energy Calculations}

All reported excitation energies are calculated using the second order n-electron valence state perturbation theory (NEVPT2) method ${ }^{102,103}$ implemented in the ORCA 
program package, ${ }^{104}$ to account for the dynamic correlation effect with the Def2-TZVPP basis set. ${ }^{96}$ Full $\pi$ valence active spaces are used in the NEVPT2 calculations. Please note that the exclusion of the $\mathrm{N}$ lone pair orbitals from the active space overestimates the vertical $E\left(S_{1}\right)$ of the $(\mathrm{CAAC})_{2}-\mathrm{C}_{2}$ dimer by $0.32 \mathrm{eV}$ in a test calculation. It is thus necessary to include them, i.e., to use the full $\pi$ valence active space. The NEVPT2 wave functions are consistent with those obtained in the MRSF-TDDFT calculations. Therefore, the geometry optimizations and excitation energy calculations are performed for the same set of states.

In the NEVPT2 calculations, states that are relevant in an excitation are averaged equally. For instance, to calculate adiabatic $E\left(S_{I}\right)$ of a molecule, NEVPT2 calculations weighting $S_{0}$ and $S_{1}$ equally are carried out at $S_{0^{-}}$and $S_{1^{-}}$-optimized structures. The difference between $E\left(S_{I}\right)$ at $S_{1}$ structure and $E\left(S_{0}\right)$ at $S_{0}$ structure (abbreviated as $\mathrm{E}\left(S_{0}\right) @ \mathrm{~S}_{0} \mathrm{~g}$ and $\left.E\left(S_{I}\right) @ S_{I} \mathrm{~g}\right)$, offset by the ZPEs of the respective structures, gives the adiabatic $\mathrm{E}\left(S_{l}\right)$. We gradually include more states in the average to ensure that proper states have been captured by the minimum average scheme. If not, then more states are included until convergences of the energies and wave functions of the interesting states are attained. To calculate an adiabatic excitation energy, we always average the same number of states in the NEVPT2 calculations at the two optimized structures.

The CAAC model adopted in this work is shown in structure 13, with all substituents on the 5-membered ring being methyls. The two $\mathrm{R}$ groups at the $\alpha \mathrm{C}$ atom are to be replaced by F atoms and trifluoromethyls to tune the electron-accepting capacity of the $\alpha \mathrm{C}$. The resultant CAAC structures are accordingly labelled as $\mathrm{CAAC}(\mathrm{F})$ and CAAC(TFM). 


\section{CHAPTER 3 CALCULATION AND DISCUSSION}

\section{Section 3.1 The Fluorination and Trifluoromethylation}

The HOMO and LUMO of CAAC in its $S_{0}$ state are shown in Figure 6(a) and (b). With the $\mathrm{N}$ as a $\pi$ donor, the $\pi$ LUMO features antibonding character between the $\mathrm{N}$ atom and the carbene $\mathrm{C}$ atom, and its energy is raised. With the lone pair electrons occupying the $\sigma \mathrm{HOMO}, \mathrm{CAAC}$ is a typical $\sigma$ donating N-heterocyclic carbene (NHC). In the CAAC $\pi \mathrm{LUMO}$, there is almost no bonding or antibonding interaction between the carbene $\mathrm{C}$ atom and the $\alpha \mathrm{C}$ atom. Quantitatively, the $\pi$ LUMO contributes -0.001 Mayer bond order ${ }^{105}$ between the two atoms. Once we replace the two methyls attached to the $\alpha \mathrm{C}$ by $\mathrm{F}$ atoms, the $\mathrm{C}-\mathrm{F} \sigma^{*}$ antibonding orbitals have lower energy and are more localized on the $\alpha \mathrm{C}$. The $\sigma^{*}$ orbitals will then become more effective in overlapping with the $p_{\pi} \mathrm{AO}$ on the carbene $\mathrm{C}$. For comparison, the $\pi$ LUMO contributes 0.091 Mayer bond order between the two $\mathrm{C}$ atoms in $\mathrm{CAAC}(\mathrm{F})$. This typical through-bond bonding interaction ${ }^{106}$ lowers the energy of the $\pi$ LUMO and stabilizes the radical with the unpaired electron in the $\pi$ orbital.

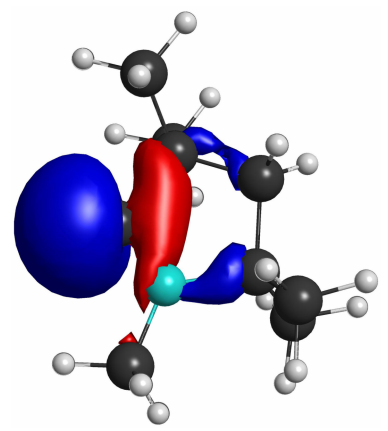

(a)

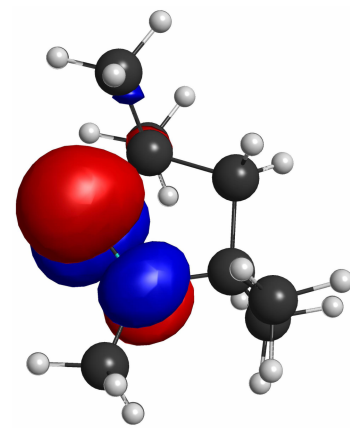

(b)

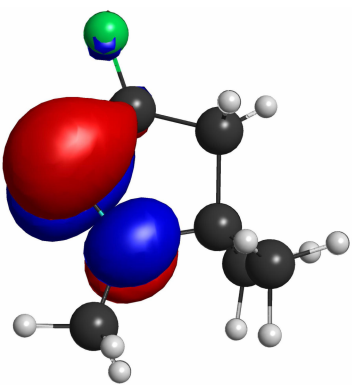

(c)

Figure 6. (a) and (b) the $\sigma \mathrm{HOMO}$ and $\pi$ LUMO of CAAC in its singlet ground state; (c) the $\pi$ LUMO of CAAC (F). 
The comparison of Figure 6(b) and (c) clearly shows that with the more electronegative $\mathrm{F}$ atoms, the $\pi$ LUMO features some bonding interaction between the carbene $\mathrm{C}$ and the $\alpha \mathrm{C}$. As a reflection of the stabilization of the $\pi \mathrm{LUMO}$, the vertical $\mathrm{E}\left(T_{1}\right)$ of $\mathrm{CAAC}(\mathrm{F})$ is calculated to be lower than that of CAAC by $0.11 \mathrm{eV}(2.61 \mathrm{vs} .2 .72$ $\mathrm{eV}$ ), and the $T_{1}$ state has one electron in each of the $\sigma$ and the $\pi$ orbital. CAAC(TFM) is in between the other two structures. The $\pi$ LUMO contributes 0.012 Mayer bond order between the carbene $\mathrm{C}$ atom and the $\alpha \mathrm{C}$ atom in $\mathrm{CAAC}(\mathrm{TFM})$, and the vertical $\mathrm{E}\left(T_{1}\right)$ is $2.67 \mathrm{eV}$. The BHHLYP HOMO-LUMO gaps are 4.57, 4.55, and $4.47 \mathrm{eV}$ for CAAC, CAAC(TFM), and CAAC(F). The gap decrease is consistent with the decrease of the vertical $\mathrm{E}\left(T_{1}\right)$ and the increase of the Mayer $\pi$ bond order between the carbene $\mathrm{C}$ atom and the $\alpha \mathrm{C}$ atom. We are aware that according to the $\sigma$ electron donor-acceptor indicator proposed by Oziminski et al., $\mathrm{CF}_{3}$ is less electronegative than $\mathrm{CH}_{3}{ }^{107}$ With no intention to question the validity of this useful indicator, we here just present a trend that is in line with the chemical intuition: the presence of the $\mathrm{F}$ atoms in $\mathrm{CF}_{3}$ makes it more electronegative than $\mathrm{CH}_{3}$, and the $\alpha \mathrm{C}$ atom with the two $\mathrm{CF}_{3}$ groups exhibits a stronger $\pi$ accepting capability. The inconsistence is partially attributed to the complexity in defining electronegativity, ${ }^{108}$ especially electronegativity of functional groups. ${ }^{109}$

There are other ways to stabilize the $\pi$ LUMO of a carbene. One straightforward way is to put a $\pi$ acceptor, e.g., a tertiary B atom, beside the carbene C. We here adopt the fluorination and trifluoromethylation strategies, with the purpose to minimize the modification of the CAAC structure. It is of critical importance to keep a quaternary $\mathrm{C}$ in the $\alpha$ position, which provides steric protection and enhances the stability of CAACs. ${ }^{84}$ 
The results presented in this work show that it is possible to stabilize the $\pi$ LUMO while maintaining a quaternary $\alpha \mathrm{C}$. While we are focusing on attaching electronegative substituents onto the $\alpha \mathrm{C}$, another way to enhance the diradical character is to attach electropositive substituent onto the $\mathrm{N}$. This substitution strategy forms a subject for future research.

\section{Section 3.2 CAAC Dimers with a $\mathrm{C}_{2}$ Spacer}

We first examine the dimers of the three types of CAAC units connected by a $\mathrm{C}_{2}$ spacer. The dimers are called $(\mathrm{CAAC})_{2}-\mathrm{C}_{2}, \quad(\mathrm{CAAC}(\mathrm{TFM}))_{2}-\mathrm{C}_{2}$, and $(\mathrm{CAAC}(\mathrm{F}))_{2}-\mathrm{C}_{2}$, respectively. These structures are of special interest as the $(\mathrm{CAAC})_{2}-\mathrm{C}_{2}$ cumulene has been isolated in good yield. ${ }^{110,111}$ It is thus desirable to investigate the (F) and (TFM) analogues with the same skeleton. We believe that the knowledge accumulated in synthesizing $(\mathrm{CAAC})_{2}-\mathrm{C}_{2}$ is helpful in synthesizing the analogues. Please note that each of the CAAC dimers is examined as a singlet fission chromophore. We need two of the CAAC dimers to give the chromophore dimer model in Figure 2.

The optimized structures of the $S_{0}, T_{1}$, and $S_{1}$ states of (CAAC) $)_{2}-\mathrm{C}_{2}$ are shown in Figure 7(a)-(c) and the calculated results are summarized in Table 2 . The $172^{\circ}$ dihedral angle $d_{\mathrm{NCCN}}$ indicates a cumulene resonance structure for this molecule. The occupancy of the lowest unoccupied natural orbital $\left(n_{L U N O}\right)$ is 0.13 , which indicates the diradical character of this molecule. ${ }^{112}$ The $n_{L U N O}$ mainly arises from the configuration with the HOMO-to-LUMO two-electron excitation configuration. The orbitals are shown in Figure 7(d) and (e). This excitation corresponds to polarizing the distributions of the two electrons in HOMO, so that when one electron occupies the $\pi$ orbital of one carbene, the 
other occupies the $\pi$ orbital of the other carbene. This suggests an increase of diradical character with the $\pi$ radical centers on the carbene $\mathrm{C}$ atoms. Meanwhile, the out-of-plane $\pi$ bonding orbital between the two central $\mathrm{C}$ atoms is strengthened due to the corresponding (anti)bonding character in the LUMO (HOMO). Therefore, the $S_{0}$ state adopts the additional diradical resonance structure shown in Figure 7(h). In consistence with the diradical resonance structure, the central $\mathrm{CC}$ bond of the cumulene moiety is shorter than the other two $(1.26$ vs. $1.33 \AA)$. Please note that these calculated bond lengths are in excellent agreement with the experimental values of 1.27 and $1.33 \AA .^{110}$ The XRD-determined bond lengths are for the single crystal of $(\mathrm{CAAC})_{2}-\mathrm{C}_{2}$ with diisopropylphenyls (Dipp) attached to the $\mathrm{N}$ atoms. The agreement reflects the accuracy of the MRSF-TDDFT structure optimization and the appropriateness of replacing the Dipp groups by methyls.

The $T_{1}$ state corresponds to the HOMO-to-LUMO single electron excitation, resulting in the pure diradical resonance structure shown in Figure 7(i). The resonance structure is consistent with the $T_{1}$ geometrical structure: the $1.24 \dot{\AA}$ short CC triple bond in the center and the almost perpendicular dihedral angle made by the two CAAC fragments $\left(d_{\mathrm{NCCN}}=97^{\circ}\right)$. The ZPE-corrected vertical and adiabatic $E\left(T_{l}\right)$ s are calculated to be 2.53 and $1.52 \mathrm{eV}$, respectively. The $1.01 \mathrm{eV}$ large reorganization energy is consistent with the significant structural change between the $S_{0}$ and $T_{I}$ states. 
Table 2. Calculated results for the three $\mathrm{C} 2$-connected dimers. All energies are given in $\mathrm{eV}$.

\begin{tabular}{cccc}
\hline & CAAC & $($ TFM $)$ & $(\mathrm{F})$ \\
\hline Adiabatic $E\left(S_{l}\right)$ & 2.15 & 2.47 & 2.33 \\
Vertical $E\left(S_{I}\right)(f)$ & $3.71(0.002)$ & $3.66(0.003)$ & $3.71(0.004)$ \\
Adiabatic $E\left(S_{2}\right)^{a}$ & 3.50 & 3.36 & 3.30 \\
Vertical $E\left(S_{2}\right)(f)$ & $3.71(0.76)$ & $3.55(0.75)$ & $3.53^{\mathrm{b}}(0.75)$ \\
Adiabatic $E\left(T_{1}\right)$ & 1.52 & 1.43 & 1.29 \\
Vertical $E\left(T_{I}\right)$ & 2.53 & 2.33 & 2.19 \\
$n_{L U N O}^{c}$ & 0.13 & 0.14 & 0.15 \\
$\Delta E_{S F}^{(1)}$ & -0.89 & -0.39 & -0.25 \\
\hline
\end{tabular}

${ }^{a}$ The $S_{2}$ structural optimizations and ZPE calculations are carried out with $\mathrm{C}_{i}$ symmetry, in which the $S_{2}$ and $S_{1}$ states belong to different irreducible representations. Therefore, $S_{1}-S_{2}$ root flipping does not occur in the course of $S_{2}$ optimizations; ${ }^{\text {b }}$ for the (F) dimer, this is actually the vertical $E\left(S_{1}\right)$. We use $S_{2}$ to label this state for comparing its energy with the energies of the truly vertical $S_{2}$ states with the same HOMO-to-LUMO excitation scheme in the CAAC dimer and the (TFM) dimer; ${ }^{c}$ calculated at $S_{0}$ structure.

The $S_{l}$ state arises from the single electron HOMO-to-LUMO+1 excitation. The planar framework remains in the $S_{l}$ optimized structure, with $\mathrm{d}_{\mathrm{NCCN}}=175^{\circ}$. At the BHHLYP/Def2-SVP level, the HOMO-LUMO gap is calculated to be $3.51 \mathrm{eV}$, not significantly lower than the $3.91 \mathrm{eV}$ HOMO-LUMO+1 gap. Please note that the orbital energies are obtained in a restricted open-shell BHHLYP calculation for the lowest quintet state at the $S_{0}$ structure, which treats HOMO-1 to LUMO+1 equally. Their orbital energies are hence comparable. The HOMO and LUMO are compact and they are both 
out-of-plane $\pi$ orbitals. They hence have a substantial spatial overlap, which results in a large exchange integral that gives a high singlet excited state with one electron in each orbital. Please note that the large orbital overlap favors a low-lying triplet state. On the contrary, the HOMO and LUMO+1 (Figure 7(f)) are perpendicular and have a smaller exchange integral that can offset their $0.4 \mathrm{eV}$ orbital gap. Consequently, the HOMO-toLUMO+1 singlet excitation is of lower energy, while the HOMO-to-LUMO excitation gives the $S_{2}$ state. The vertical excitation energies of the two singlet states are close. With ZPE corrections, they are both $3.71 \mathrm{eV}$.
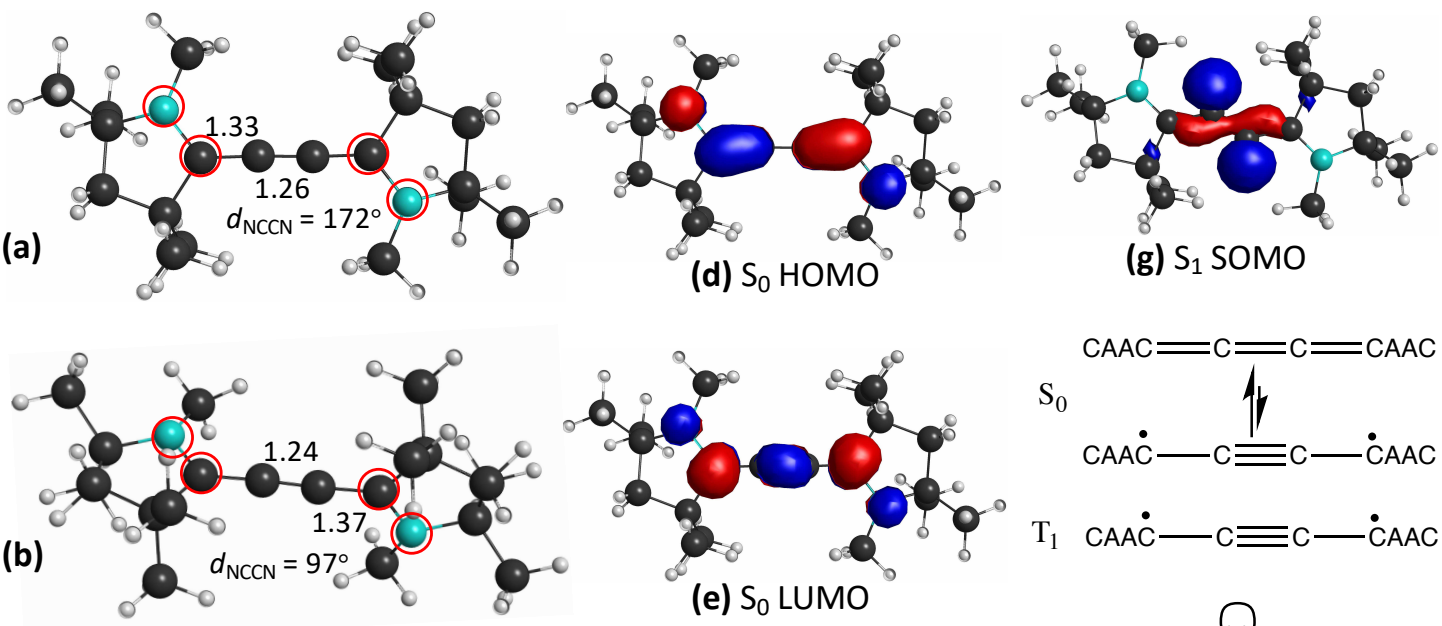

(d) $\mathrm{S}_{0} \mathrm{HOMO}$

(g) $\mathrm{S}_{1} \mathrm{SOMO}$

(e) $\mathrm{S}_{0}$ LUMO
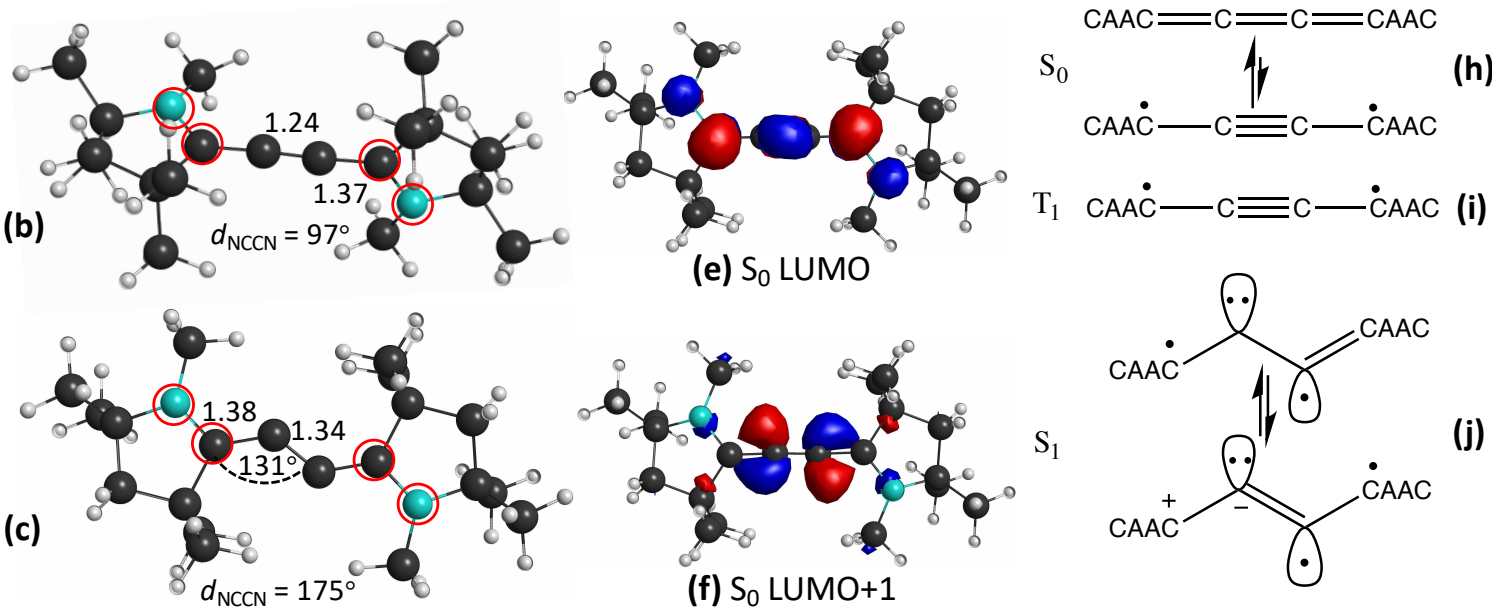<smiles>CC(=O)OC1=CC(C)C(C)=C1C(C)=O</smiles>

Figure 7. The optimized structures of the (a) $S_{0}$, (b) $T_{1}$, and (c) $S_{1}$ states of (CAAC) $)_{2}-\mathrm{C}_{2}$; (d)-(g) frontier orbitals of the structures of the denoted states; (h)-(i) typical resonance structures of the three states. Some important structural parameters are shown in panels (a)-(c), where the bond lengths are given in $\AA$ and the four atoms that define the dihedral angles are highlighted by red circles. In panel (i), only one set of resonance structures are shown for the $S_{I}$ state. There is another set which includes the symmetry images of the shown structures. 
We cannot reproduce the non-ZPE-corrected $2.88 \mathrm{eV}$ vertical $E\left(S_{1}\right)$ of $(\mathrm{CAAC})_{2}$ $\mathrm{C}_{2}$ reported in Ref. 83. We took the structure from the Supportive Information of the paper and averaged the lowest five singlet states (employing the same state average scheme in the reference) in performing NEVPT2 calculation for the structure. The vertical $E\left(S_{l}\right)$ is calculated to be $3.13 \mathrm{eV}$, with $f=0.58$. This $f$ is close to the 0.6 reported in Ref. 83. This $S_{1}$ state corresponds to the HOMO-to-LUMO excitation. The HOMO-toLUMO+1 excitation gives the $S_{2}$ state, with the non-ZPE-corrected vertical $E\left(S_{1}\right)=3.60$ $\mathrm{eV}$. There are two factors that contribute to the differences between the results in Ref. 83 and ours. (1) Different active spaces are used. A smaller 4 electrons in 4 orbitals active space was used in Ref. 83, while the full valence $\pi$ active space with 8 orbitals and 10 electrons are employed in the present work. As mentioned in Chapter 2, the calculated excitation energies of the $\mathrm{C}_{2}$-connected dimers (and the $\mathrm{C}_{4}$-connected dimers too) are highly sensitive to the active space, varying by tenths of eV. (2) Different dimer structures were used. The 1.33 and 1.26 A CC bond lengths demonstrated in Figure 7(a) are in good agreement with the experimental values. The corresponding bond lengths of the structure used in Ref. 83 are 1.44 and $1.18 \AA$.

The $S_{1}$-optimized structure features an in-plane bending of the cumulene moiety, resulting in the $131^{\circ} \angle \mathrm{CCC}$ shown in Figure $7(\mathrm{c})$. The in-plane $\pi^{*}$ antibonding LUMO+1 at the $S_{0}$ structure has evolved to the nonbonding singly occupied molecular orbital (SOMO) at the $S_{1}$ structure (Figure $7(\mathrm{~g})$ ). Correspondingly, the in-plane $\pi$ bonding orbital has also evolved to a similar nonbonding orbital. There is thus little in-plane $\pi$ bonding interaction between the two central C atoms. The $S_{l}$ state adopts the resonance structures 
shown in Figure 7(j), with three electrons distributed in the two in-plane $s p^{2}$ hybridized non-bonding orbitals. The ZPE-corrected vertical and adiabatic $E\left(S_{I}\right)$ s are calculated to be 3.71 and $2.15 \mathrm{eV}$. The significant structural change in the $S_{l}$ state is commensurate with the $1.56 \mathrm{eV}$ large reorganization energy. The perpendicular HOMO and LUMO+1 give the 0.002 small oscillator strength $(f)$ for the vertical $S_{0}$-to- $S_{l}$ excitation. The $2.15 \mathrm{eV}$ adiabatic $E\left(S_{l}\right)$ and the $3.71 \mathrm{eV}$ vertical $E\left(S_{l}\right)$ are in fair agreement with the UV/Vis spectrum of the dimer in $\mathrm{CH}_{2} \mathrm{Cl}_{2}$ solution. ${ }^{111}$ The spectrum consists of a structureless band, whose low energy edge is at $2.32 \mathrm{eV}(535 \mathrm{~nm})$ and with very low intensity. The intensity of the band increases sharply as the absorption energy increases to $3.65 \mathrm{eV}$ ( 340 $\mathrm{nm}$ ), beyond which the absorption was not recorded. The $340 \mathrm{~nm}$ absorption is so intense that it must involve the excitation to $S_{2}$, whose adiabatic and vertical excitation energies are 3.50 and $3.71 \mathrm{eV}$ (354 and $334 \mathrm{~nm}$ ), respectively, and $f=0.76$. Please note that the agreement between the calculated excitation energies and the UV/Vis spectrum corroborates the accuracy of our calculation methods.

With the $C_{i}$ symmetry of the dimer in crystal, ${ }^{11,110}$ the HOMO-to-LUMO+1 excitation to the $S_{I}$ state is symmetry forbidden. In solution, the dimer can also adopt $C_{2}$ symmetry, as the $C_{2}$ configuration is only higher by $0.002 \mathrm{eV}$ (estimated at the NEVPT2 level) than the $C_{i}$ configuration. Although the transition is now not symmetry-forbidden, as mentioned above, the small $f=0.002$ arises from the transition between two perpendicular $\pi$ orbitals. Despite the small (strictly zero) oscillator strength in $C_{2}\left(C_{i}\right)$ symmetry, the transition may be turned on by vibrations, and one possible vibrational mode is the torsional motion of the CAAC units about the $\mathrm{C}_{2}$ spacer. Such an out-ofplane vibration can induce mixing of the out-of-plane LUMO and the in-plane LUMO+1, 
and hence turns on the HOMO-to-LUMO+1 transition. According to our MRSF-TDDFT calculation, the frequency of this mode is only $40 \mathrm{~cm}^{-1}$ low. Out-of-plane wagging and rocking modes of the CAAC units with respect to the spacer are of similarly low frequencies. These vibrations can be thermally activated. Overall, the structure of the dimer is not rigid and vibration-induced $S_{0}$-to- $S_{1}$ transition is possible. Other than the symmetry-breaking vibrations, the $S_{0}$-to- $S_{I}$ transition intensity may also be enhanced by the surrounding $\mathrm{CH}_{2} \mathrm{Cl}_{2}$ polar solvent molecules. The mixing of the perpendicular LUMO and LUMO +1 can be induced by an electric dipole pointing in a direction between them. The conjecture of the vibration- and solvent-induced $S_{0}$-to- $S_{l}$ transition is consistent with the low intensity of the absorption at $2.32 \mathrm{eV}(535 \mathrm{~nm})$.

The excited states of $(\mathrm{CAAC}(\mathrm{TFM}))_{2}-\mathrm{C}_{2}$ and $(\mathrm{CAAC}(\mathrm{F}))_{2}-\mathrm{C}_{2}$ feature similar excitation schemes and take similar resonance and geometrical structures for those states. Their higher diradical characters are reflected by their lower vertical $E\left(T_{1}\right) \mathrm{s}$ of 2.33 and $2.19 \mathrm{eV}$ and their larger $n_{\mathrm{LUNO}}$ of 0.14 and 0.15 . The order of the diradical character follows the expectation based on the stabilization of the CAAC $\pi$ LUMO by the trifluoromethylation and fluorination at the $\alpha \mathrm{C}$. With structural reorganization, the adiabatic $E\left(T_{I}\right) \mathrm{s}$ are calculated to be 1.43 and $1.29 \mathrm{eV}$.

The $S_{l}$ state has little to do with the diradical character, and the ZPE-corrected vertical $E\left(S_{l}\right) \mathrm{s}$ of the (TFM) and (F) dimers are calculated to be 3.66 and $3.71 \mathrm{eV}$, which are close to the $3.71 \mathrm{eV}$ of the unsubstituted structure. After the $S_{1}$ structures are optimized, the adiabatic $E\left(S_{I}\right) \mathrm{s}$ are 2.47 and $2.33 \mathrm{eV}$, larger than the $2.15 \mathrm{eV}$ of the unsubstituted structure. The higher $E\left(S_{I}\right)$ s leave more room for the substituted dimers to satisfy Eq. 1. Please note that at the $S_{0}$ structure of the (F) dimer, the HOMO-to- 
LUMO+1 excitation requires slightly higher energy than the HOMO-to-LUMO excitation (3.71 vs. $3.53 \mathrm{eV}$, also see the $b$ note in Table 2). The HOMO-to-LUMO+1 excited state becomes lower in energy in the course of the $S_{l}$ structural optimization and gives the adiabatic $S_{l}$ state. For this reason and also the convenience to compare with the results of (CAAC)- $\mathrm{C}_{2}$ and the (TFM) dimer, we still label the HOMO-to-LUMO+1 excitation at the $S_{0}$ structure the $S_{1}$ state, while the HOMO-to-LUMO excitation the $S_{2}$ state.

With the ZPE-corrected adiabatic energies, $\Delta E_{S F}^{(1)}=E\left(S_{1}\right)-2 E\left(T_{1}\right)=-0.89,-0.39$, and $-0.25 \mathrm{eV}$ for $(\mathrm{CAAC})_{2}-\mathrm{C}_{2}$, the (TFM), and the (F) dimers. None of them meets the primary criterion Eq. 1 for SF chromophores, although the trend is in the right direction with the TFM and F substitutions. In addition to the energy mismatches, these molecules have another disadvantage as SF chromophores. Their $>3.6 \mathrm{eV}$ vertical $E\left(S_{1}\right) \mathrm{s}$ are in the UV region, in which the solar spectrum has low photon flux density. And the $S_{1}$ states have negligible oscillator strengths. The $S_{2}$ states involve the HOMO-to-LUMO excitation and their oscillator strengths are more substantial. Their vertical excitation energies are deeper in the UV region (except for the $(\mathrm{F})$ dimer, whose vertical $E\left(S_{2}\right)$ is at the border of the UV and Vis regions). Overall, the three CAAC dimers do not have good absorption of the solar spectrum. The importance of structural reorganization of the excited states is clearly shown in this series of calculations. If we compare the vertical $E\left(S_{1}\right) \mathrm{s}$ and the adiabatic $E\left(T_{1}\right) \mathrm{s}$, all three molecules satisfy Eq. 1. The $>1.19 \mathrm{eV}$ large reorganization energies of the $S_{1}$ states, however, reverse the signs of the respective $\Delta E_{S F}^{(1)} \mathrm{S}$ 


\section{Section 3.3 CAAC Dimers with a $\mathrm{C}_{4}$ Spacer}

The HOMO and LUMO in Figure 7(d) and (e) show that the $\pi$ LUMOs of the CAAC units have (anti)bonding interactions with the LUMO (HOMO) of the $\mathrm{C}_{2}$ spacer. Without these interactions, each of the CAAC $\pi$ LUMO would hold an unpaired electron in the dimer, and the dimer would be a pure diradical. The bonding and antibonding interactions open up the HOMO-LUMO gap and reduce the diradical character, so that the dimer does not satisfy Eq. 1. A longer spacer, with its attenuated HOMO and LUMO amplitudes at the connecting sites with the CAAC units, is likely to reduce the bonding/antibonding interactions and increase the diradical character, making the dimer satisfy Eq. 1. Following this logic, we increase the length of the spacer from $\mathrm{C}_{2}$ to $\mathrm{C}_{4}$. The calculated results of the $\mathrm{C}_{4}$-connected dimers are summarized in Table 3 .

The $S_{0}, T_{1}$, and $S_{1}$ structures of $(\mathrm{CAAC})_{2}-\mathrm{C}_{4}$ are shown in Figure 8(a)-(c), which share geometrical features with the $\mathrm{C}_{2}$ analogues. This is reasonable given the similarity in electronic structure of cumulenes with different lengths. While the HOMO in Figure 8(d) shows bonding interaction between the CAAC fragments and the spacer, the LUMO+1 in Figure 8(f), which corresponds to the LUMO in Figure 7(e), shows largely nonbonding character. The LUMO and LUMO+1 are almost degenerate, with the 2.53

and $2.62 \mathrm{eV}$ HOMO-LUMO and HOMO-LUMO+1 gaps at the BHHLYP level of calculation. 

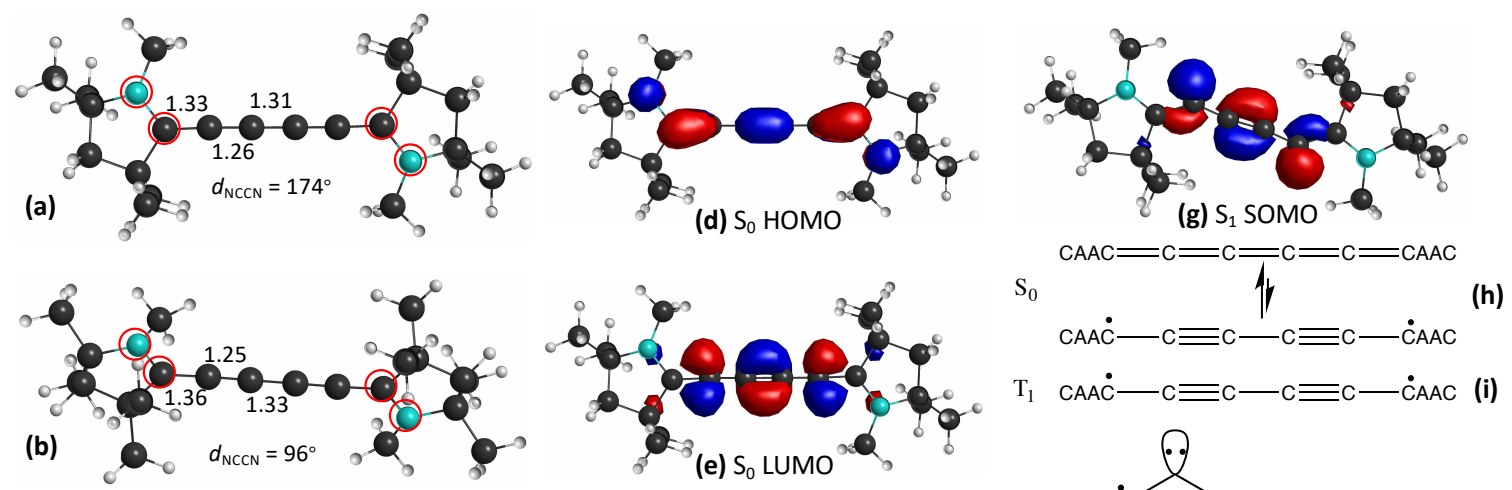

CAAC- $\mathrm{C} \equiv \mathrm{C}-\mathrm{C} \equiv \mathrm{C}-\dot{\mathrm{C}} \mathrm{AAC}$

$\mathrm{T}_{1} \quad \mathrm{CAAC}-\mathrm{C} \equiv \mathrm{C}-\mathrm{C} \equiv \mathrm{C}$ - $\dot{\mathrm{C} A A C}$ (i)

(c)
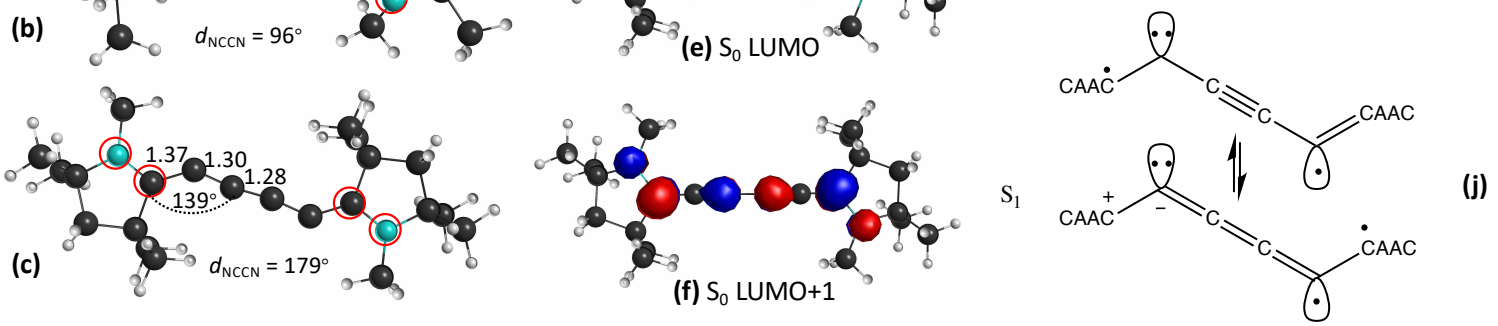

Figure 8 . The optimized structures of the (a) $S_{0}$, (b) $T_{1}$, and (c) $S_{1}$ states of $(\mathrm{CAAC})_{2}-\mathrm{C}_{4}$; (d)-(g) frontier orbitals of the structures of the denoted states; (h)-(j) typical resonance structures of the three states. Some important structural parameters are shown in panels (a)-(c), where the bond lengths are given in $\AA$ and the four atoms that define the dihedral angles are highlighted by red circles. In panel $(\mathrm{j})$, only one set of resonance structures are shown for the $S_{l}$ state. There is another set which includes the symmetry images of the shown structures.

The $T_{1}$ and $S_{1}$ states involve the same excitations as in the $\mathrm{C}_{2}$-connected dimers, except that the LUMO and $\mathrm{LUMO}+1$ of the $\mathrm{C}_{4}$-connected dimer correspond to the LUMO +1 and LUMO of the $\mathrm{C}_{2}$ analogue. The vertical $E\left(T_{1}\right) \mathrm{s}$ are $1.80,1.65$, and $1.58 \mathrm{eV}$ for $(\mathrm{CAAC})_{2}-\mathrm{C}_{4},(\mathrm{CAAC}(\mathrm{TFM}))_{2}-\mathrm{C}_{4}$, and $(\mathrm{CAAC}(\mathrm{F}))_{2}-\mathrm{C}_{4}$, respectively, and the ground state $n_{\mathrm{LUNO}} \mathrm{S}$ are $0.19,0.20$, and 0.21 . Both sets of data indicate the stronger diradical characters brought by the $\mathrm{C}_{4}$ spacer, and brought by the trifluoromethylation and fluorination. The pure diradical character of the $T_{1}$ state results in the structural reorganization to the perpendicular arrangement of the CAAC fragments as shown in Figure 8(b), along with a $0.74 \mathrm{eV}$ substantial reorganization energy. 
In the $S_{l}$ state the antibonding LUMO is occupied and evolves to the SOMO at the $S_{1}$ structure (Figure $8(\mathrm{~g})$ ) following the bending of the $\mathrm{C}_{6}$ cumulene fragment. The SOMO exhibits nonbonding character at the two ends of the spacer. The $0.79 \mathrm{eV} S_{1}$ reorganization energy is similar to the $T_{1}$ analogue. The structures, orbitals, and excitation schemes of the $\mathrm{C}_{4}$-connected dimers are similar to those of the $\mathrm{C}_{2}$-connected dimers. We thus assign the similar resonance structures as shown in Figure 8(h)-(j) to the three states of $(\mathrm{CAAC})_{2}-\mathrm{C}_{4}$. The structures and orbitals of the (TFM) and $(\mathrm{F})$ dimers are similar to those of the unsubstituted dimer and are hence not shown in Figure 8. 
Table 3. Calculated results for the three $\mathrm{C} 4$-connected dimers. All energies are given in $\mathrm{eV}$.

\begin{tabular}{cccc}
\hline & CAAC & $(\mathrm{TFM})$ & $(\mathrm{F})$ \\
\hline Adiabatic $E\left(S_{l}\right)$ & 1.65 & 1.89 & 1.87 \\
Vertical $E\left(S_{l}\right)(f)$ & $2.44(0.0001)$ & $2.50(0.001)$ & $2.56(0.001)$ \\
Vertical $E\left(S_{2}\right)^{a}(f)$ & $3.33(0.71)$ & $3.19(0.61)$ & $3.16(0.62)$ \\
Adiabatic $E\left(T_{1}\right)$ & 1.06 & 0.98 & 0.91 \\
Vertical $E\left(T_{I}\right)$ & 1.80 & 1.65 & 1.58 \\
$E\left(T_{I}\right) @ S_{I} g$ & 1.40 & 1.82 & 1.65 \\
$n_{\mathrm{LUNO}}^{b}$ & 0.19 & 0.20 & 0.21 \\
$\lambda^{c}$ & 1.08 & 1.51 & 1.41 \\
Barrier $^{d}$ & 0.56 & 0.41 & 0.33 \\
$\Delta \mathrm{E}_{\mathrm{SF}}^{(1)}$ & -0.47 & -0.07 & 0.05 \\
\hline
\end{tabular}

${ }^{a}$ Without ZPE correction; ${ }^{b}$ calculated at $S_{0}$ structure; ${ }^{c}$ reorganization energies for $S_{0} S_{I} \rightarrow T_{1} T_{1} ;{ }^{d}$ barrier for $S_{0} S_{I} \rightarrow T_{1} T_{1}$.

The $S_{1}$ and $T_{1}$ energies of the three $\mathrm{C}_{4}$-connected dimers, both vertical and adiabatic, are lower than the $\mathrm{C}_{2}$ counterparts. This is consistent with the smaller HOMOto-LUMO and HOMO-to-LUMO+1 gaps brought by the longer length of the molecules. With the adiabatic $E\left(S_{l}\right) \mathrm{s}$ and $E\left(T_{1}\right) \mathrm{s}, \Delta E_{S F}^{(1)}=-0.47,-0.07$, and $0.05 \mathrm{eV}$. The (TFM) dimer is close to satisfy and the (F) dimer satisfies Eq. 1. The $0.07 \mathrm{eV} \mathrm{SF}$ endoergicity of the (TFM) dimer can be overcome by thermal activation. Even for the unsubstituted structure, the subtraction results in a less negative value than the $\mathrm{C}_{2}$ analogue. With the 
smaller orbital gaps, the vertical excitation energies of the lowest light-absorbing singlet excited state $E\left(S_{2}\right) \mathrm{s}$ are $3.33,3.19$, and $3.16 \mathrm{eV}$ for the three $\mathrm{C}_{4}$-connected dimers, all smaller than the corresponding energies of the $\mathrm{C}_{2}$-connected dimers. The $S_{2}$ state is dominated by the HOMO-to-LUMO+1 single electron excitation and has large oscillator strength: $f=0.71,0.61$, and 0.62 for the three dimers. Those absorptions occur in the visible region of the solar spectrum, which has higher photon flux density than the UV region for the $\mathrm{C}_{2}$-connected dimers. The $E\left(S_{2}\right) \mathrm{s}$ above do not contain ZPE corrections. They are higher than the corresponding $E\left(S_{l}\right) \mathrm{s}$ by $0.5 \mathrm{eV}$, and the ZPE corrections would not alter the $S_{1}-S_{2}$ energy ordering. We hence do not optimize the $S_{2}$ structures and calculate their ZPEs. On the contrary, the vertical $E\left(S_{1}\right)$ and $E\left(S_{2}\right) \mathrm{s}$ of the $\mathrm{C}_{2}$-connected dimers are close and it is necessary to include ZPE corrections to clarify the energy ordering.

Overall, the improvement by replacing the $\mathrm{C}_{2}$ spacer by the $\mathrm{C}_{4}$ is evident. However, it is too early to conclude that $(\mathrm{CAAC}(\mathrm{TFM}))_{2}-\mathrm{C}_{4}$ and $(\mathrm{CAAC}(\mathrm{F}))_{2}-\mathrm{C}_{4}$ are good candidates for SF chromophores. The substantial structural reorganizations in the $S_{0}$-to- $S_{1}$ and $S_{0}$-to- $T_{1}$ excitations and the significant structural differences between the $S_{l^{-}}$ and $T_{1}$-optimized structures imply large reorganization energies $(\lambda \mathrm{s})$ for the $S_{0} S_{1} \rightarrow T_{1} T_{1}$ processes in the two dimers. $\lambda$ can be approximated as

$$
\begin{aligned}
\lambda & =E\left(T_{1}\right) @ S_{0} g+E\left(T_{1}\right) @ S_{1} g-2 E\left(T_{1}\right) @ T_{1} g \\
& =E\left(T_{1}\right) @ S_{0} g+E\left(T_{1}\right) @ S_{1} g-E\left(S_{l}\right) @ S_{I} g+\Delta E_{S F}^{(1)} .
\end{aligned}
$$

Please note that $E\left(S_{0}\right) @ S_{0} g$ has been defined to be the 0 reference. For the $\mathrm{C}_{4}$ connected dimers, the $T_{1}$ states at the $S_{1}$ structures have the same occupation scheme as the corresponding $S_{1}$ states. The different $T_{1}$ occupation schemes compared to those in the 
$S_{0}$ structures are a reasonable result of the stabilization of the $S_{I}$ SOMO shown in Figure $8(\mathrm{~g})$. With the excitation energies reported in Table 3, is calculated to be $1.08,1.51$ and $1.41 \mathrm{eV}$ for the three $\mathrm{C}_{4}$-connected dimers. According to the Marcus Theory ${ }^{113}$, the barrier for the fission can be estimated as $\frac{\left(\lambda-\Delta E_{S F}^{(1)}\right)^{2}}{4 \lambda}$. For the three dimers, the barriers are estimated to be $0.56,0.41$ and $0.33 \mathrm{eV}$, respectively. They are fairly high for a photoinduced process such as SF, recalling the limited life times of the $S_{I}$ states. Although the (TFM) and (F) dimers satisfy the thermodynamics criterion Eq. 1, SFs are not likely to occur in those systems due to kinetics unfavorability. Please note that only the Marcus Theory is used to estimate the SF barriers in this work, without any intention to claim that the fissions, if they occur, follow the perturbation mechanism of the theory. It requires excited states dynamics simulations to explore the time scales of singlet fission in those chromophore candidates. And these simulations are beyond the scope of this research. Therefore, we cannot conclude on the validity of the Marcus Theory in the possible SF of the chromophore candidates studied in this work.

The large barriers arise from the significant structural reorganizations in the $T_{l}$ states from the $S_{0}$ and $S_{1}$ structures to the $T_{1}$ structure. The $\mathrm{C}_{2}$ and $\mathrm{C}_{4}$ spacers are cylindrically symmetric, and so are their valence $\pi$ systems. The cylindrical $\pi$ systems allow the two CAAC units to take a perpendicular configuration along the spacers, so that the SOMOs of the $T_{l}$ states are perpendicular to each other and change from bonding and antibonding orbitals in the $S_{0}$ structure to nonbonding orbitals in the $T_{1}$ structure (Figure $9)$. 
(a)
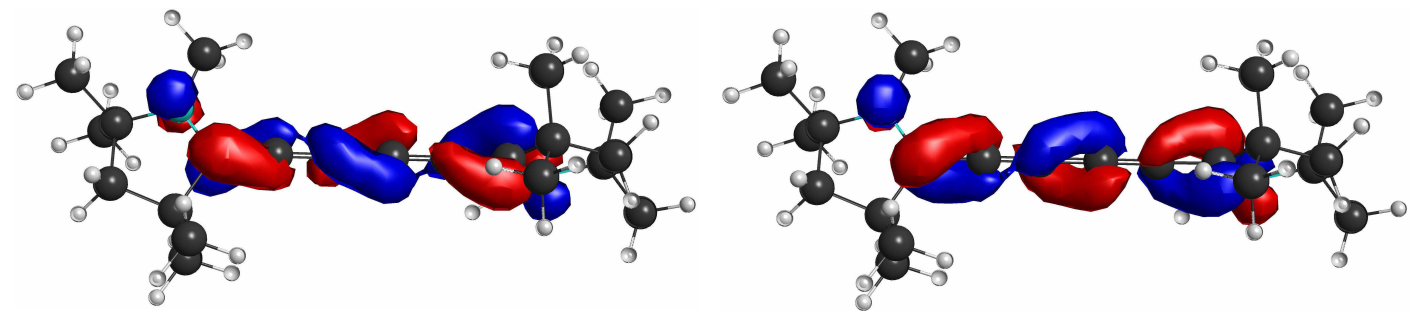

(b)
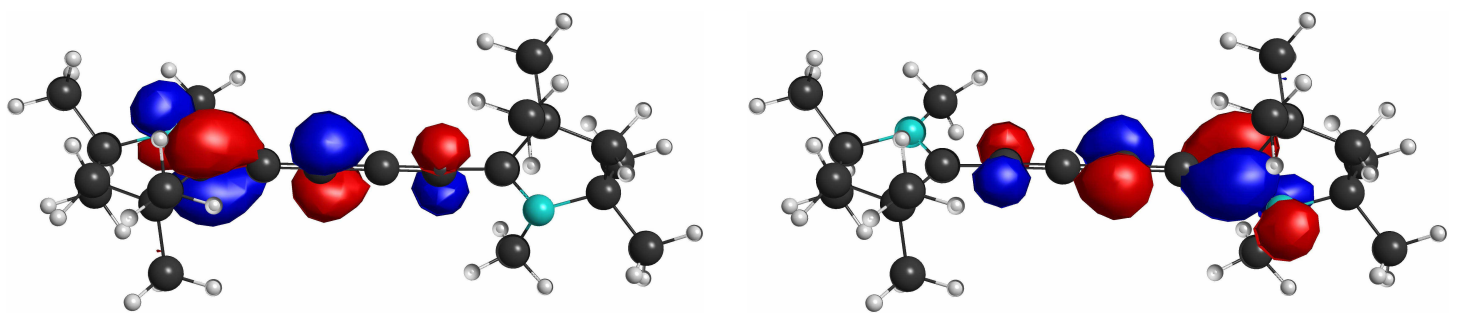

Figure 9. (a) The two singly occupied molecular orbitals $\mathrm{SOMO}_{1}$ and $\mathrm{SOMO}_{2}$ in the $T_{1}$ state of $(\mathrm{CAAC})_{2}-\mathrm{C}_{4}$ and (b) the corresponding localized orbitals obtained from $1 / \sqrt{2}\left(\mathrm{SOMO}_{1} \pm \mathrm{SOMO}_{2}\right)$, which are perpendicular $\pi$ orbitals.

Our calculation shows that each of the SOMOs in Figure 9(a) contributes $\leq 0.06$ Mayer bond order between adjacent $\mathrm{C}$ atoms along the central linear fragment of the molecule. The nonbonding nature of the SOMOs is evident. These nonbonding SOMOs lower $E\left(T_{1}\right)$ along the coplanar-to-perpendicular structural reorganization, just as in the case of the triplet ethylene. Please note that by "coplanar" and "perpendicular", we only consider the configuration of the $\mathrm{N}$, the carbene $\mathrm{C}$, and the $\alpha \mathrm{C}$ in the two CAAC units, and the spacer. The significant $T_{1}$ structural reorganization has two opposite effects for $(\mathrm{CAAC}(\mathrm{TFM}))_{2}-\mathrm{C}_{4}$ and $(\mathrm{CAAC}(\mathrm{F}))_{2}-\mathrm{C}_{4}:(1)$ it lowers their adiabatic $E\left(T_{1}\right)$ s so that Eq. 1 is satisfied or closed to be satisfied; (2) it raises the reorganization energies and also the barriers in SF, so that the two dimers are unlikely to undergo SF. The cylindrical $\pi$ systems consist of perpendicular (out-of-plane and in-plane) $\pi$ orbitals in the $S_{0}$ structures. The $S_{1}$ states arise from electron transition from out-of-plane orbital to inplane orbital. The antibonding interaction of the singly occupied in-plane $\pi^{*}$ orbital is 
mitigated by the in-plane bending of the $S_{l}$ structure, which enhances the nonbonding character of the orbital. In short, the cylindrical $\pi$ systems of the $\mathrm{C}_{2}$ and $\mathrm{C}_{4}$ spacers are responsible for their large $S_{1}$ and $T_{1}$ reorganization energies.

Knowing the kinetics unfavorability, we do not need to consider Eq. 2 for the $\mathrm{C}_{4}$ connected dimers. we mention in passing that the optimizations of $T_{2}$ structures of the $\mathrm{C}_{2}$ and $\mathrm{C}_{4}$ - connected dimers failed to converge. This is because the $T_{2}$ states have the same ${ }^{3} \mathrm{~B}$ symmetry as the $T_{1}$ states and the two states exchange their characters (i.e., root flipping) during the optimization. The $T_{2}$ states of the $\mathrm{C}_{2}$-connected dimers correspond to the HOMO-to-LUMO+1 excitation, and $\mathrm{C}_{4}$ analogues correspond to the HOMO-1-toLUMO excitation. The HOMO- 1 of $(\mathrm{CAAC})_{2}-\mathrm{C}_{4}$ is shown in

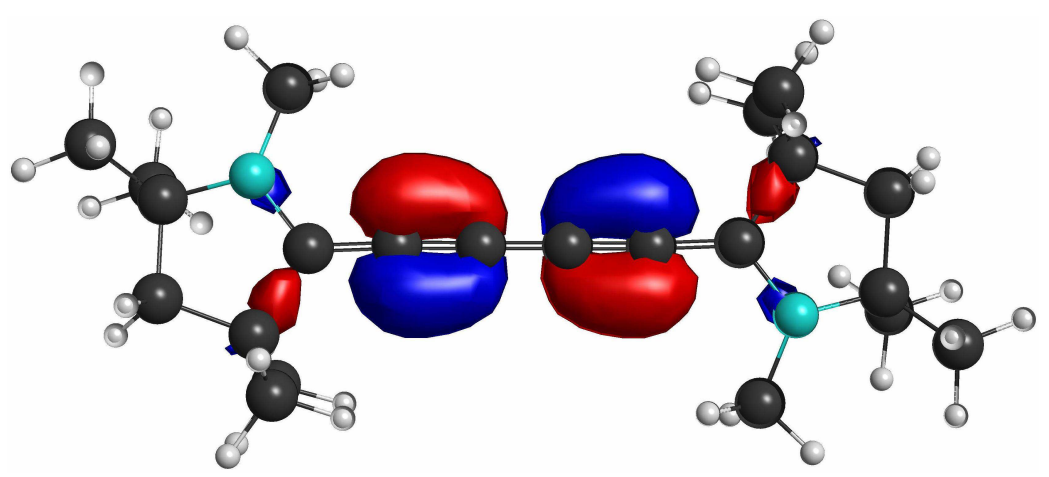

Figure 10. HOMO-1 of (CAAC $)_{2}-\mathrm{C}_{4}$. 


\section{Section 3.4 CAAC Dimers with a Para-Phenylene Spacer}

To avoid the problem induced by the cylindrical system of the spacer, we select the $p$-phenylene (abbreviated as $\mathrm{Ph}$ ) as the spacer to connect two CAAC moieties. Unlike the sequence of presentation in the previous sections, we first focus on the results of $(\mathrm{CAAC}(\mathrm{F}))_{2}-\mathrm{Ph}$ and $(\mathrm{CAAC}(\mathrm{TFM}))_{2}-\mathrm{Ph}$. This is because the optimization of the $S_{1}$ state of (CAAC $)_{2}-\mathrm{Ph}$ failed.

The $S_{0^{-}}, T_{1^{-}}$, and $S_{1^{-}}$optimized structures of $(\mathrm{CAAC}(\mathrm{F}))_{2}-\mathrm{Ph}$ are shown in Figure 11(a), (b), and (c). The calculated results are summarized in Table 4. They look more similar to each other than the $\mathrm{C}_{2}$ and $\mathrm{C}_{4}$ analogues. Especially, the key dihedral angle changes by up to $20^{\circ}$ only. Here, we choose the dihedral angle made by the CAAC unit and the phenyl spacer $\left(d_{\mathrm{NCCC}}\right)$ to indicate the coplanarity of the central fragment of the dimer. The smaller changes in the structure are consistent with the smaller $S_{l}$ and $T_{1}$ reorganization energies, which are 0.20 and $0.54 \mathrm{eV}$, respectively, vs. the 0.69 and 0.67 $\mathrm{eV}$ of $(\mathrm{CAAC}(\mathrm{F}))_{2}-\mathrm{C}_{4}$. The $(\mathrm{TFM})$ dimer shares similar structures with $(\mathrm{CAAC}(\mathrm{F}))_{2}-\mathrm{Ph}$ and thus also has smaller $S_{1}$ and $T_{1}$ reorganization energies than its $\mathrm{C}_{4}$ counterpart. The replacement of $\mathrm{C}_{4}$ by $p$-phenylene does point in the right direction.

The $1.53 \mathrm{eV}$ vertical $E\left(T_{I}\right)$ of $(\mathrm{CAAC}(\mathrm{F}))_{2}-\mathrm{Ph}$ is similar to the $1.58 \mathrm{eV}$ of $(\mathrm{CAAC}(\mathrm{F}))_{2}-\mathrm{C}_{4}$. This suggests similar diradical characters of the two dimers. The 0.18 $n_{\mathrm{LUNO}}$ of $(\mathrm{CAAC}(\mathrm{F}))_{2}-\mathrm{Ph}$, however, suggests a lower diradical character. This inconsistence is attributed to the difference in the active spaces in use. For $(\mathrm{CAAC}(\mathrm{F}))_{2}$ $\mathrm{C}_{4}$, the in-plane $\pi$ electrons can be excited and contribute to the higher $0.21 n_{\mathrm{LUNO}}$. Similar in-plane $\pi$ electrons are not present in $(\mathrm{CAAC})_{2}-\mathrm{Ph}$. It is more reasonable to compare $n_{\mathrm{LUNO}}$ s from calculations with comparable active spaces, e.g., those of the $\mathrm{C}_{2^{-}}$ 
and $\mathrm{C}_{4}$-connected dimers. We hence take the vertical $E\left(T_{1}\right)$ as a more reliable indicator to compare the diradical characters of $(\mathrm{CAAC}(\mathrm{F}))_{2}-\mathrm{Ph}$ and $(\mathrm{CAAC}(\mathrm{F}))_{2}-\mathrm{C}_{4}$.
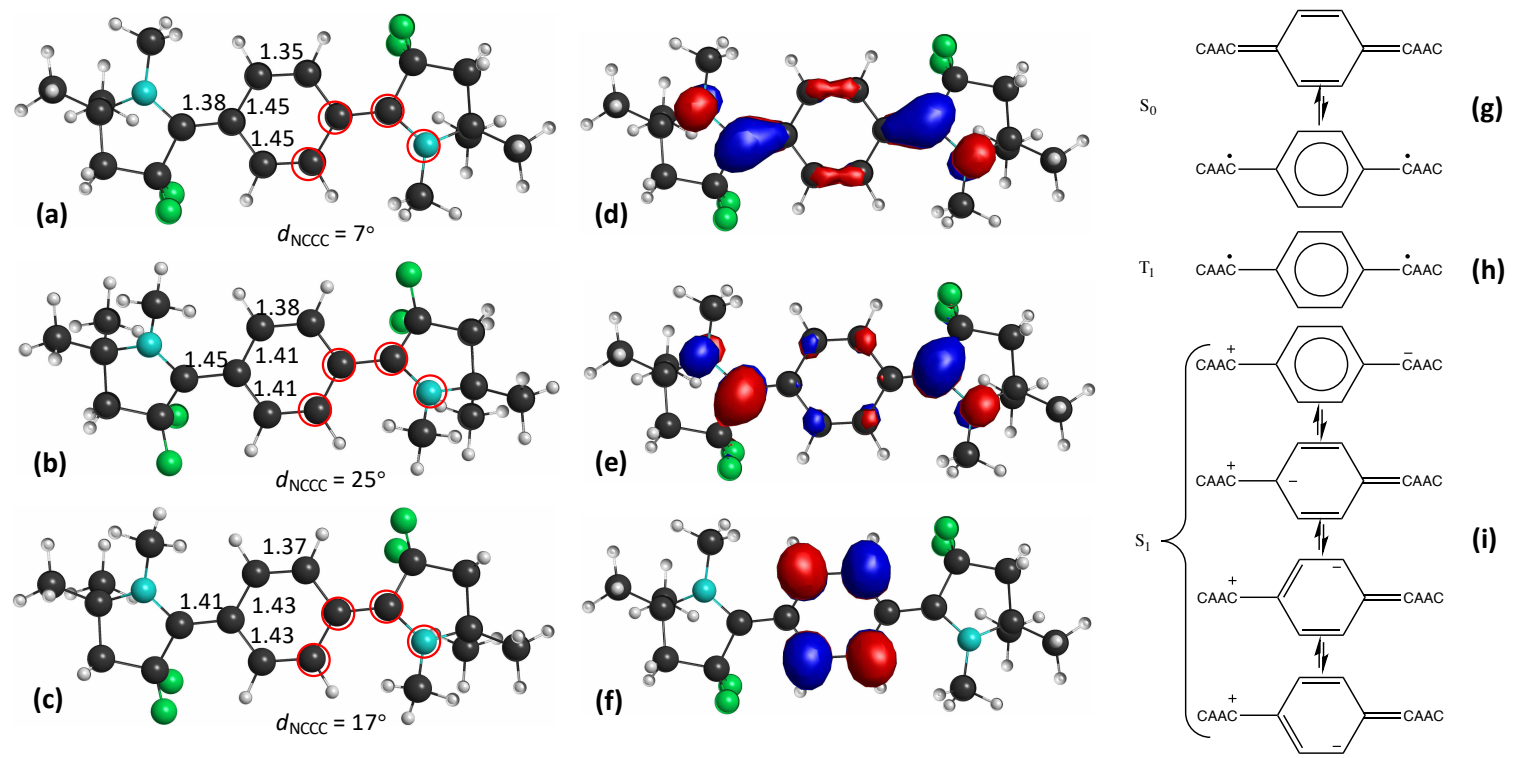

Figure 11. The optimized structures of the (a) $S_{0}$, (b) $T_{1}$, and (c) $S_{1}$ states of (CAAC(F)) $2_{2}$ $\mathrm{Ph}$; (d)-(f) frontier orbitals of the structures of the $S_{0}$ state; (g)-(i) typical resonance structures of the three states. Some important structural parameters are shown in panels (a)-(c), where the bond lengths are given in $\AA$ and the four atoms that define the dihedral angles are highlighted by red circles. In panel (i), only some resonance structures are shown for the $S_{1}$ state. The others include those with the + and - charges being swapped and those with the symmetry images.

$(\mathrm{CAAC}(\mathrm{F}))_{2}-\mathrm{Ph}$ satisfies Eq. 1, with $\Delta E_{S F}^{(1)}=-0.62 \mathrm{eV}$. Both the $S_{1}$ and $T_{1}$ states correspond to the HOMO-to-LUMO excitation, and the HOMO and LUMO are shown in Figure 11(d) and (e). The HOMO (LUMO) arises from (anti)bonding interaction between the LUMO (HOMO) of the phenylene spacer and the two $\pi$ orbitals of the carbenes. $(\mathrm{CAAC}(\mathrm{F}))_{2}-\mathrm{Ph}$ bears a typical $p$-quinodimethane core structure, and its diradical character is associated to the $S_{0}$ resonance structure with an aromatic phenylene (see the $S_{0}$ resonance structures in Figure $\left.11(\mathrm{~g})\right)$. The $S_{0}$ state is dominated by the resonance 
structure with alternating $\mathrm{CC}$ single and double bonds, as reflected by the bond lengths shown in Figure 11(a). The $T_{1}$ state simply takes the diradical resonance structure associated with the phenylene spacer in its aromatic form. Correspondingly, the $\mathrm{CC}$ bond lengths of the phenylene are more equalized in this state, and the $\mathrm{CC}$ bonds that connect the CAAC units and the spacer are elongated from 1.38 to $1.45 \AA$ (i.e., from double bonds between $s p^{2} \mathrm{C}$ atoms to single bonds).

Table 4. Calculated results for the two substituted Ph-connected dimers. All energies are given in $\mathrm{eV}$

\begin{tabular}{cccc}
\hline & CAAC & $(\mathrm{TFM})$ & $(\mathrm{F})$ \\
\hline Adiabatic $E\left(S_{I}\right)$ & $2.45^{a}$ & 2.31 & 2.60 \\
Vertical $E\left(S_{l}\right)(f)$ & $2.59(0.83)$ & $2.45(0.70)$ & $2.70(0.85)$ \\
Adiabatic $E\left(T_{1}\right)$ & $1.24^{a}$ & 0.94 & 0.99 \\
Vertical $E\left(T_{1}\right)$ & 1.63 & 1.41 & 1.53 \\
$E\left(T_{1}\right) @ S_{I} g$ & $1.34^{\mathrm{a}}$ & 1.13 & 1.20 \\
Adiabatic $E\left(T_{2}\right)$ & 2.68 & 3.18 & 3.07 \\
$n_{L U N O}^{b}$ & 0.16 & 0.20 & 0.18 \\
$\lambda^{c}$ & 0.49 & 0.66 & 0.75 \\
Barrier $^{d}$ & 0.14 & 0.02 & 0.02 \\
$\Delta \mathrm{E}_{\mathrm{SF}}^{(1)}$ & 0.03 & 0.43 & 0.62 \\
$\Delta \mathrm{E}_{\mathrm{SF}}^{(2)}$ & 0.20 & 1.30 & 1.09
\end{tabular}

${ }^{a}$ The adiabatic $E\left(S_{l}\right)$ and $E\left(T_{1}\right) @ S_{I} g$ of the CAAC dimer is for the $S_{l}$-optimized structure with $C_{2}$ symmetry, which has an imaginary frequency. ${ }^{b}$ calculated at $S_{0}$ structure; ${ }^{c}$ reorganization energies for $S_{0} S_{1} \rightarrow T_{1} T_{1} ;{ }^{d}$ barrier for $S_{0} S_{I} \rightarrow T_{1} T_{l}$. 
It is well known that the HOMO-to-LUMO one-electron singlet transition results in a charge resonance state with one of the localized orbitals in Figure 12 being doubly occupied while the other being empty. ${ }^{114}$ The charge resonance structures shown in Figure 11(i) correspond to that an electron is depleted from the CAAC on the left side, which contributes the most to the left localized orbital in Figure 12, and the electron is deposited in the $\mathrm{C}$ sites on the spacer and the other CAAC that contribute to the right localized orbital in the figure. The charge-resonance structures with the backward electron transfers and symmetry images of all these structures also contribute to the $\mathrm{S}_{1}$ state, but are not shown in Figure 11(i).

The $T_{2}$ state of $(\mathrm{CAAC}(\mathrm{F}))_{2}-\mathrm{Ph}$ corresponds to the HOMO-to-LUMO+1 excitation (see Figure 11(f) for LUMO+1). The ZPE-corrected adiabatic $E\left(T_{2}\right)$ is calculated to be $3.07 \mathrm{eV}$, giving $\Delta E_{S F}^{(2)}=E\left(T_{2}\right)-2 E\left(T_{1}\right)=1.09 \mathrm{eV}$, satisfying Eq. 2. With $E\left(T_{1}\right) @ S_{1} g=1.20 \mathrm{eV}$, the reorganization energy for the $S_{0} S_{l} \rightarrow T_{1} T_{1}$ fission is estimated to be $0.75 \mathrm{eV}$, and the barrier is estimated to be $0.02 \mathrm{eV}$. The less substantial structural reorganizations of the $S_{1}$ and $T_{1}$ states lead to the half smaller compared to $(\mathrm{CAAC}(\mathrm{F}))_{2}-$ $\mathrm{C}_{4}$. 

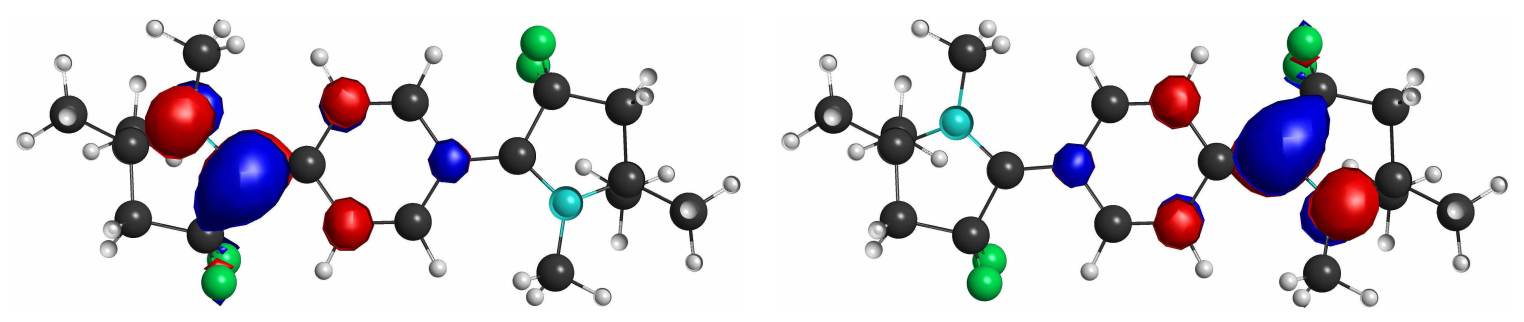

Figure 12. Localized orbitals $(\mathrm{CAAC}(\mathrm{F}))_{2}-\mathrm{Ph}$ obtained from $1 / \sqrt{ } 2(\mathrm{HOMO} \pm \mathrm{LUMO})$

The smaller $\lambda$ together with the large positive $\Delta E_{S F}^{(1)}$ result in the essentially barrier-less SF kinetics. The vertical $E\left(S_{l}\right)$ is $2.70 \mathrm{eV}$ with $f=0.85$. It corresponds to an intense absorption at the visible region at the solar spectrum that has fairly high photon flux density. Another advantage of $(\mathrm{CAAC}(\mathrm{F}))_{2}-\mathrm{Ph}$ is its $0.99 \mathrm{eV}$ adiabatic $E\left(T_{1}\right)$, which is close to the $\sim 1 \mathrm{eV}$ optimal band gap that maximizes efficiency of a SF-based photovoltaics device. ${ }^{1,10}$ With all these advantages, $(\mathrm{CAAC}(\mathrm{F}))_{2}-\mathrm{Ph}$ is a good candidate for SF chromophore.

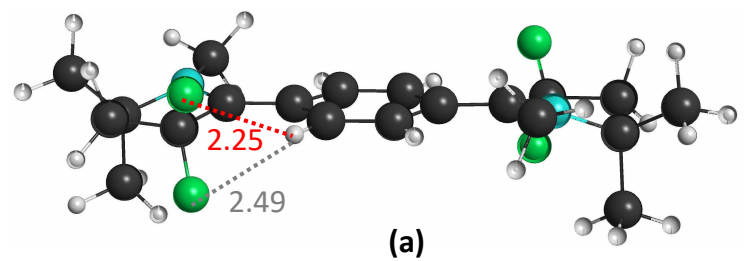

(a)

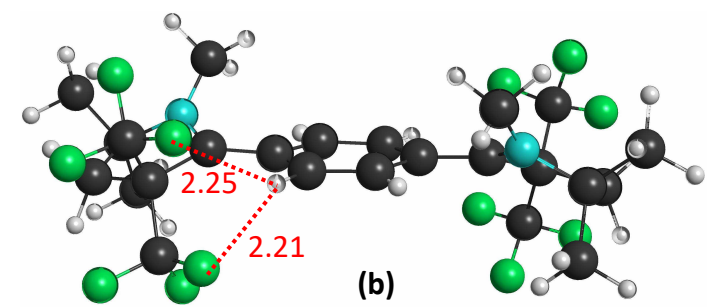

(b)

Figure 13. Hydrogen bonds of $\mathrm{F}$ atoms in (a) $(\mathrm{CAAC}(\mathrm{F}))_{2}-\mathrm{Ph}$ and (b) $(\mathrm{CAAC}(\mathrm{TFM}))_{2}-$ $\mathrm{Ph}$. The highlighted internuclear distances are given in $\AA$. The red dashed lines indicate F $\cdots$ H hydrogen bonds. 
All those advantages are shared by the (TFM) dimer, which is thus another good candidate. It is interesting that the (TFM) dimer appears to have a higher diradical character than the (F) dimer, indicated by its higher $n_{\mathrm{LUNO}}=0.20$ and lower vertical $E\left(T_{l}\right)$ $=1.41 \mathrm{eV}$. This is inconsistent with the expectation based on the electronegativities of TFM and F. We attribute this anomaly to the F $\cdots \mathrm{H}$ hydrogen bonds between the $\mathrm{F}$ atoms on the CAAC units and the $\mathrm{H}$ atoms on the spacer. One of the two highlighted F-H distances shown in Figure 13(a) for the (F) dimer is shorter than the $2.26 \AA$ typical F $\cdots \mathrm{H}$ bond distance between a $\mathrm{C}\left(s p^{3}\right)-\mathrm{F}$ and and a $\mathrm{C}\left(s p^{2}\right)-\mathrm{H} .{ }^{115}$ There is certainly $\mathrm{F} \cdots \mathrm{H}$ bonding interaction between the CAAC units and the spacer. As shown in Figure 13(b), there are two F-H distances between one CAAC unit and the spacer that are shorter than $2.26 \AA$. There are thus twice more F $\cdots \mathrm{H}$ hydrogen bonds in the (TFM) dimer. This is reasonable as some F atoms of the TFM groups are more oriented towards the spacer. The stronger F $\cdots \mathrm{H}$ interaction in the (TFM) dimer requires the CAAC unit to adopt a more preferable configuration for the hydrogen bonds. This results in the larger dihedral angle, $\mathrm{d}_{\mathrm{NCCC}}=$ $20^{\circ}$, in $(\mathrm{CAAC}(\mathrm{TFM}))_{2}-\mathrm{Ph}$. The weaker $\mathrm{F} \cdots \mathrm{H}$ interaction allows a smaller $\mathrm{d}_{\mathrm{NCCC}}=7^{\circ}$ in the (F) dimer, which favors $\pi$ bonding interaction between the CAAC units and the spacer. The larger dihedral angle in $(\mathrm{CAAC}(\mathrm{TFM}))_{2}-\mathrm{Ph}$ results in less overlap between the carbene $\pi$ orbitals and the LUMO and HOMO of the spacer, and thus the higher diradical character of $(\mathrm{CAAC}(\mathrm{TFM}))_{2}-\mathrm{Ph}$. 

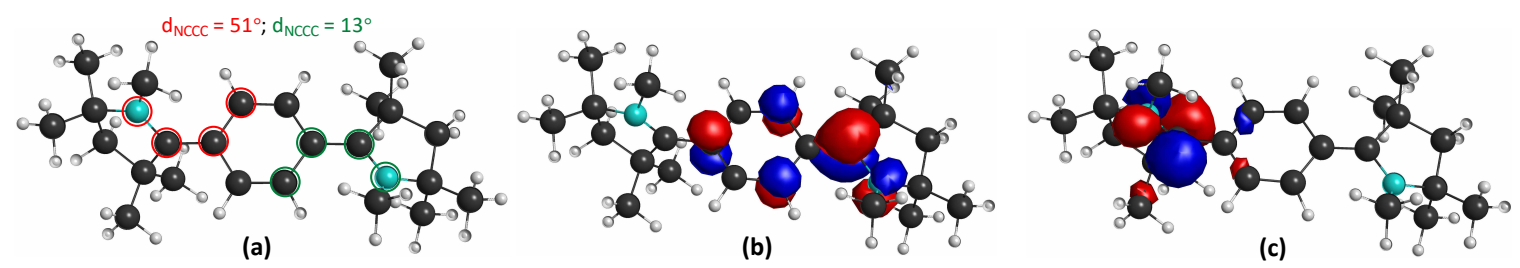

Figure 14. A representative structure (a) obtained during the optimization of the $S_{1}$ structure of (CAAC) $)_{2}-\mathrm{Ph}$ and its (b) HOMO and (c) LUMO.

The 0.43 and $0.62 \mathrm{eV} \Delta E_{S F}^{(1)} \mathrm{s}$ of $(\mathrm{CAAC}(\mathrm{TFM}))_{2}-\mathrm{Ph}$ and $(\mathrm{CAAC}(\mathrm{F}))_{2}-\mathrm{Ph}$ seem large and imply energy waste and slow SF. However, they are not large when compared with the $0.58 \mathrm{eV} \Delta E_{S F}^{(1)}$ of molecular pentacene. The $\Delta E_{S F}^{(1)}$ of this most intensely studied SF chromophore decreases to $0.11 \mathrm{eV}$ in solid state, due to the significant reduction of its adiabatic $E\left(S_{l}\right)$ from 2.31 to $1.86 \mathrm{eV}$. This red-shift of $E\left(S_{l}\right)$ in changing from molecules to their crystals is common for organic molecules and is attributed to the Coulomb interaction between neighboring molecules and Davydov splitting. ${ }^{116}$ The seemingly large $\Delta E_{S F}^{(1)} \mathrm{s}$ of the two dimeric molecules have reserved some room for the red-shifts, so that the dimers in solids can still satisfy Eq. 1. Without running solid state calculations, we cannot predict the red-shifts. However, we note that $\mathrm{E}\left(S_{l}\right) \approx 2 \mathrm{E}\left(T_{1}\right)$ may not be an essential requirement for isolated molecules in designing SF chromophores. The approximate isoergicity relation is more relevant for SF in solution or intramolecular SF.

The unsubstituted dimer $(\mathrm{CAAC})_{2}-\mathrm{Ph}$, if all its structures of the four relevant states maintained $\mathrm{C}_{2}$ symmetry, would also be a possible candidate for SF chromophore. The energies in Table 4 of this dimer are obtained using the $\mathrm{C}_{2}$ structures. These energies give $\Delta E_{S F}^{(1)}=-0.03 \mathrm{eV}, \Delta E_{S F}^{(2)}=0.20 \mathrm{eV}$, and a $0.14 \mathrm{eV} \mathrm{SF}$ barrier. The non-ZPE-corrected 
vertical $\mathrm{E}\left(S_{I}\right)$ and vertical $\mathrm{E}\left(T_{1}\right)$ are calculated to be 2.76 and $1.72 \mathrm{eV}$, which are in good agreement with the 2.74 and $1.70 \mathrm{eV}$ calculated values reported in Ref. 83. The small negative $\Delta E_{S F}^{(1)}$ can be overcome by thermal activation and the barrier is surmountable. However, the $S_{1}$ structure optimized with $C_{2}$ symmetry features an imaginary frequency with a symmetry-breaking mode. The optimization following this mode leads to structure with one CAAC unit being significantly non-coplanar with the spacer. One such representative structure is shown in Figure 14(a), with the increased $d_{\mathrm{NCCC}}$ angle marked in red color and the other in green color. The HOMO and LUMO of this structure are shown in Figure 14(b) and (c).

At this structure, the $S_{l}$ state corresponds to an open-shell singlet with one electron in each of the HOMO and LUMO, while the $S_{0}$ state corresponds to double occupation of the HOMO. The $S_{0}-S_{1}$ gap is only $0.24 \mathrm{eV}$. The two states are about to exchange their characters, and they do a few optimization steps later. The $S_{l}$ structural optimization thus failed. Taking $E\left(S_{0}\right) @ S_{0} g$ as the reference, the non-ZPE-corrected E $\left(S_{l}\right)$ at this structure is calculated to be $2.18 \mathrm{eV}$, which is substantially less than $2 E\left(T_{1}\right)=2 \times$ $1.35=2.70 \mathrm{eV}$. Please note that this $E\left(T_{l}\right)$ does not contain ZPE correction. It is comparable with the non-ZPE-corrected $E\left(S_{I}\right)$. Therefore, even if we can locate the minimum on the $S_{l}$ potential energy surface, $(\mathrm{CAAC})_{2}-\mathrm{Ph}$ would not satisfy Eq. 1 . The $S_{0}-S_{I}$ root flipping in the structural optimization implies that the $S_{I}$ would rapidly decay to the $S_{0}$ state radiationlessly. The short-lived $S_{1}$ also disfavors SF.

The reorganization of the $S_{I}$ structure into this asymmetric configuration can be rationalized as follows. As mentioned above, the singlet open-shell with one electron in each of the delocalized HOMO and LUMO in Figure 11(d) and (e) correspond to a 
charge resonance closed-shell state, with two electrons in one of the localized orbitals in Figure 12 and none in the other. Such a state features long distance charge transfer between the two CAAA $\pi$ orbitals (e.g., the first resonance structure in figure 11(i)), which is energy costly. By rotating one CAAC unit out of the plane of the phenylene spacer, the overlap is lost and the delocalized HOMO and LUMO have evolved to the less delocalized HOMO and the localized LUMO in Figure 14s(b) and (c). The singlet open-shell with one electron in each of these resultant orbitals does not involve the long distance charge transfer between the CAAC units. The energy of the $S_{l}$ state is hence lower. This tendency to lower the $S_{l}$ energy by rotating one CAAC unit out of the plane of the spacer must also be applicable in $\left(\mathrm{CAAC}(\mathrm{TFM})_{2}-\mathrm{Ph}\right.$ and $(\mathrm{CAAC}(\mathrm{F}))_{2}-\mathrm{Ph}$. However, the F $\cdots \mathrm{H}$ bonds shown in Figure 13 prevent this distortion from happening. 


\section{CHAPTER 4 CONCLUSION}

With the objective to design new singlet fission chromophores, we employ MRSF-TDDFT and NEVPT2 methods to investigate a series of cyclic (alkyl)(amino)carbenes (CAAC) dimers. In the dimers, the CAAC units are connected by $\mathrm{C}_{2}, \mathrm{C}_{4}$, and $p$-phenylene spacers, and they are substituted by trifluoromethyls and fluorine atoms at their $\alpha \mathrm{C}$ position. The trifluoromethylation and fluorination are found to stabilize the $\pi$ LUMO of CAAC, consequently enhance the diradical character for the CAAC dimers, and make them closer to satisfy or satisfy the $E\left(S_{l}\right) \geq 2 E\left(T_{I}\right)$ primary energy condition for singlet fission. Meanwhile, the $\alpha \mathrm{C}$ remains being a quaternary $\mathrm{C}$. The two substitution schemes will find more use beyond the context of singlet fission, as they can functionalize CAAC in other fields of optoelectronics. The spacers mainly have two effects: (1) the frontier orbitals of a longer spacer $\left(\mathrm{C}_{4}\right.$ vs. $\left.\mathrm{C}_{2}\right)$ are more attenuated and have smaller $\pi$ overlaps with the orbital of the CAAC units, leading to higher diradical characters of the dimers; (2) cylindrically symmetric spacers $\mathrm{C}_{2}$ and $\mathrm{C}_{4}$ allow significant structural reorganizations in the excited states, which potentially lead to endoergicity and high barrier for singlet fission. With both the attenuated frontier orbitals and noncylindrical symmetry of the spacer, $p$-phenylene-connected dimers with $\mathrm{CF}_{3}$ and $\mathrm{F}$ at the $\alpha \mathrm{C}$ position are found to be promising candidates for both thermodynamically and kinetically favorable singlet fission. The presence of the $\mathrm{F}$ atoms results in hydrogen bonds between the CAAC units and the spacer. The hydrogen bonds prevent a SFunfavorable structural distortion in the $S_{1}$ state, which occurs in the (CAAC) $)_{2}-\mathrm{Ph}$ dimer.

Other than proposing the two chromophore candidates, this study demonstrates the importance of considering structural reorganizations in designing singlet fission 
chromophores. Considering vertical $E\left(S_{1}\right) \mathrm{s}$ and adiabatic $E\left(T_{1}\right) \mathrm{s}$, all three $\mathrm{C}_{2}$-connected dimers satisfy $E\left(S_{I}\right) \geq 2 E\left(T_{I}\right)$. The significant $S_{I}$ structural reorganizations reverse the signs of $E\left(S_{l}\right)-2 E\left(T_{1}\right)$. The structural reorganizations of the $S_{l}$ and $T_{l}$ states also lead to high barriers for the singlet fission of the $\mathrm{C}_{4}$-connected trifluoromethylated and the fluorinated dimers. The two dimers, although they satisfy (or close to satisfy) $E\left(S_{l}\right) \geq$ $2 E\left(T_{1}\right)$, are hence not good candidates for singlet fission chromophores. It is a common practice to only consider vertical excitation energies in a quick survey of a large amount of structures in designing singlet fission chromophores. However, it is necessary to consider structural reorganizations of the excited states in order to more conclusively judge whether a molecule is a good candidate. 


\section{Reference}

1. Smith, M. B. \& Michl, J. Singlet Fission. Chem. Rev. 110, 6891-6936 (2010).

2. Smith, M. B. \& Michl, J. Recent Advances in Singlet Fission. Annu. Rev. Phys. Chem. 64, 361-386 (2013).

3. Thorsmølle, V. K. et al. Morphology Effectively Controls Singlet-Triplet Exciton Relaxation and Charge Transport in Organic Semiconductors. Phys. Rev. Lett. 102, 17401 (2009).

4. Wilson, M. W. B. et al. Ultrafast Dynamics of Exciton Fission in Polycrystalline Pentacene. J. Am. Chem. Soc. 133, 11830-11833 (2011).

5. Marciniak, H. et al. Ultrafast Exciton Relaxation in Microcrystalline Pentacene Films. Phys. Rev. Lett. 99, 176402 (2007).

6. Chan, W.-L. et al. Observing the Multiexciton State in Singlet Fission and Ensuing Ultrafast Multielectron Transfer. Science (80-. ). 334, 1541 LP - 1545 (2011).

7. Chan, W.-L., Ligges, M. \& Zhu, X.-Y. The energy barrier in singlet fission can be overcome through coherent coupling and entropic gain. Nat. Chem. 4, 840 (2012).

8. Bearpark, M. J. et al. The Azulene S1 State Decays via a Conical Intersection: A CASSCF Study with MMVB Dynamics. J. Am. Chem. Soc. 118, 169-175 (1996).

9. Shockley, W. \& Queisser, H. J. Detailed balance limit of efficiency of p-n junction solar cells. J. Appl. Phys. 32, 510-519 (1961).

10. Hanna, M. C. \& Nozik, A. J. Solar conversion efficiency of photovoltaic and photoelectrolysis cells with carrier multiplication absorbers. J. Appl. Phys. 100, 74510 (2006).

11. Lee, J., Jadhav, P. \& Baldo, M. A. High efficiency organic multilayer photodetectors based on singlet exciton fission. Appl. Phys. Lett. 95, 33301 (2009).

12. Congreve, D. N. et al. External Quantum Efficiency Above $100 \%$ in a SingletExciton-Fission-Based Organic Photovoltaic Cell. Science (80-. ). 340, 334 LP 337 (2013).

13. Thompson, N. J., Congreve, D. N., Goldberg, D., Menon, V. M. \& Baldo, M. A. Slow light enhanced singlet exciton fission solar cells with a $126 \%$ yield of electrons per photon. Appl. Phys. Lett. 103, 263302 (2013).

14. Pazos-Outón, L. M. et al. A Silicon-Singlet Fission Tandem Solar Cell Exceeding 100\% External Quantum Efficiency with High Spectral Stability. ACS Energy Lett. 2, 476-480 (2017).

15. Sanders, S. N. et al. Intramolecular Singlet Fission in Oligoacene Heterodimers. Angew. Chemie Int. Ed. 55, 3373-3377 (2016).

16. Xia, J. et al. Singlet Fission: Progress and Prospects in Solar Cells. Adv. Mater. 29, 1601652 (2017).

17. Piland, G. B., Burdett, J. J., Dillon, R. J. \& Bardeen, C. J. Singlet Fission: From Coherences to Kinetics. J. Phys. Chem. Lett. 5, 2312-2319 (2014).

18. Scholes, G. D. Correlated Pair States Formed by Singlet Fission and ExcitonExciton Annihilation. J. Phys. Chem. A 119, 12699-12705 (2015).

19. Tayebjee, M. J. Y. et al. Quintet multiexciton dynamics in singlet fission. Nat. Phys. 13, 182 (2016).

20. Basel, B. S. et al. Unified model for singlet fission within a non-conjugated covalent pentacene dimer. Nat. Commun. 8, 15171 (2017). 
21. Tempelaar, R. \& Reichman, D. R. Vibronic exciton theory of singlet fission. I. Linear absorption and the anatomy of the correlated triplet pair state. J. Chem. Phys. 146, 174703 (2017).

22. Kolomeisky, A. B., Feng, X. \& Krylov, A. I. A Simple Kinetic Model for Singlet Fission: A Role of Electronic and Entropic Contributions to Macroscopic Rates. $J$. Phys. Chem. C 118, 5188-5195 (2014).

23. Peierls, R. E. Quantum Theory of Solids. (Clarendon Press, 1996).

24. Tamura, H., Huix-Rotllant, M., Burghardt, I., Olivier, Y. \& Beljonne, D. FirstPrinciples Quantum Dynamics of Singlet Fission: Coherent versus Thermally Activated Mechanisms Governed by Molecular \$lensuremath $\{\mathrm{pi}\} \$$ Stacking. Phys. Rev. Lett. 115, 107401 (2015).

25. Singh, S., Jones, W. J., Siebrand, W., Stoicheff, B. P. \& Schneider, W. G. Laser Generation of Excitons and Fluorescence in Anthracene Crystals. J. Chem. Phys. 42, 330-342 (1965).

26. Swenberg, C. E. \& Stacy, W. T. Bimolecular radiationless transitions in crystalline tetracene. Chem. Phys. Lett. 2, 327-328 (1968).

27. Geacintov, N., Pope, M. \& Vogel, F. Effect of Magnetic Field on the Fluorescence of Tetracene Crystals: Exciton Fission. Phys. Rev. Lett. 22, 593-596 (1969).

28. Groff, R. P., Avakian, P. \& Merrifield, R. E. Coexistence of Exciton Fission and Fusion in Tetracene Crystals. Phys. Rev. B 1, 815-817 (1970).

29. Swenberg, C. E., van Metter, R. \& Ratner, M. Comments on exciton fission and electron spin resonance in tetracene single crystals. Chem. Phys. Lett. 16, 482-485 (1972).

30. Burgos, J., Pope, M., Swenberg, C. E. \& Alfano, R. R. Heterofission in pentacenedoped tetracene single crystals. Phys. status solidi 83, 249-256 (1977).

31. Tomkiewicz, Y., Groff, R. P. \& Avakian, P. Spectroscopic Approach to Energetics of Exciton Fission and Fusion in Tetracene Crystals. J. Chem. Phys. 54, 45044507 (1971).

32. Burdett, J. J., Müller, A. M., Gosztola, D. \& Bardeen, C. J. Excited state dynamics in solid and monomeric tetracene: The roles of superradiance and exciton fission. J. Chem. Phys. 133, 144506 (2010).

33. Burdett, J. J., Gosztola, D. \& Bardeen, C. J. The dependence of singlet exciton relaxation on excitation density and temperature in polycrystalline tetracene thin films: Kinetic evidence for a dark intermediate state and implications for singlet fission. J. Chem. Phys. 135, 214508 (2011).

34. Burdett, J. J. \& Bardeen, C. J. Quantum Beats in Crystalline Tetracene Delayed Fluorescence Due to Triplet Pair Coherences Produced by Direct Singlet Fission. J. Am. Chem. Soc. 134, 8597-8607 (2012).

35. Roberts, S. T. et al. Efficient Singlet Fission Discovered in a Disordered Acene Film. J. Am. Chem. Soc. 134, 6388-6400 (2012).

36. Mastron, J. N., Roberts, S. T., McAnally, R. E., Thompson, M. E. \& Bradforth, S. E. Aqueous Colloidal Acene Nanoparticles: A New Platform for Studying Singlet Fission. J. Phys. Chem. B 117, 15519-15526 (2013).

37. Najafov, H., Lee, B., Zhou, Q., Feldman, L. C. \& Podzorov, V. Observation of long-range exciton diffusion in highly ordered organic semiconductors. Nat. Mater. 9, 938 (2010). 
38. Ryasnyanskiy, A. \& Biaggio, I. Triplet exciton dynamics in rubrene single crystals. Phys. Rev. B 84, 193203 (2011).

39. Jankus, V. et al. Competition between polaron pair formation and singlet fission observed in amorphous rubrene films. Phys. Rev. B 87, 224202 (2013).

40. Rao, A. et al. Exciton Fission and Charge Generation via Triplet Excitons in Pentacene/C60 Bilayers. J. Am. Chem. Soc. 132, 12698-12703 (2010).

41. Rao, A., Wilson, M. W. B., Albert-Seifried, S., Di Pietro, R. \& Friend, R. H. Photophysics of pentacene thin films: The role of exciton fission and heating effects. Phys. Rev. B 84, 195411 (2011).

42. Ramanan, C., Smeigh, A. L., Anthony, J. E., Marks, T. J. \& Wasielewski, M. R. Competition between Singlet Fission and Charge Separation in Solution-Processed Blend Films of 6,13-Bis(triisopropylsilylethynyl)pentacene with StericallyEncumbered Perylene-3,4:9,10-bis(dicarboximide)s. J. Am. Chem. Soc. 134, 386397 (2012).

43. Walker, B. J., Musser, A. J., Beljonne, D. \& Friend, R. H. Singlet exciton fission in solution. Nat. Chem. 5, 1019 (2013).

44. Eaton, S. W. et al. Singlet Exciton Fission in Polycrystalline Thin Films of a SlipStacked Perylenediimide. J. Am. Chem. Soc. 135, 14701-14712 (2013).

45. Busby, E. et al. A design strategy for intramolecular singlet fission mediated by charge-transfer states in donor-acceptor organic materials. Nat. Mater. 14, 426 (2015).

46. Kraabel, B., Hulin, D., Aslangul, C., Lapersonne-Meyer, C. \& Schott, M. Triplet exciton generation, transport and relaxation in isolated polydiacetylene chains: Subpicosecond pump-probe experiments. Chem. Phys. 227, 83-98 (1998).

47. Gradinaru, C. C. et al. An unusual pathway of excitation energy deactivation in carotenoids: Singlet-to-triplet conversion on an ultrafast timescale in a photosynthetic antenna. Proc. Natl. Acad. Sci. 98, 2364 LP - 2369 (2001).

48. Wang, C., Schlamadinger, D. E., Desai, V. \& Tauber, M. J. Triplet Excitons of Carotenoids Formed by Singlet Fission in a Membrane. ChemPhysChem 12, 28912894 (2011).

49. Wang, C. \& Tauber, M. J. High-Yield Singlet Fission in a Zeaxanthin Aggregate Observed by Picosecond Resonance Raman Spectroscopy. J. Am. Chem. Soc. 132, 13988-13991 (2010).

50. Musser, A. J. et al. Activated Singlet Exciton Fission in a Semiconducting Polymer. J. Am. Chem. Soc. 135, 12747-12754 (2013).

51. Kawata, S. et al. Singlet Fission of Non-polycyclic Aromatic Molecules in Organic Photovoltaics. Adv. Mater. 28, 1585-1590 (2016).

52. Kasha, M. Characterization of electronic transitions in complex molecules. Discuss. Faraday Soc. 9, 14-19 (1950).

53. Lower, S. K. \& El-Sayed, M. A. The triplet state and molecular electronic processes in organic molecules. Chem. Rev. 66, 199-241 (1966).

54. El-Sayed, M. A. Triplet state. Its radiative and nonradiative properties. Acc. Chem. Res. 1, 8-16 (1968).

55. Paci, I. et al. Singlet Fission for Dye-Sensitized Solar Cells: Can a Suitable Sensitizer Be Found? J. Am. Chem. Soc. 128, 16546-16553 (2006).

56. Bendikov, M. et al. Oligoacenes: theoretical prediction of open-shell singlet 
diradical ground states. J. Am. Chem. Soc. 126, 7416-7417 (2004).

57. Hachmann, J., Dorando, J. J., Avilés, M. \& Chan, G. K.-L. The radical character of the acenes: A density matrix renormalization group study. J. Chem. Phys. 127, 134309 (2007).

58. Yang, Y., Davidson, E. R. \& Yang, W. Nature of ground and electronic excited states of higher acenes. Proc. Natl. Acad. Sci. 113, E5098-E5107 (2016).

59. Pariser, R. \& Parr, R. G. A Semi-Empirical Theory of the Electronic Spectra and Electronic Structure of Complex Unsaturated Molecules. I. J. Chem. Phys. 21, 466-471 (1953).

60. Pople, J. A. Electron interaction in unsaturated hydrocarbons. Trans. Faraday Soc. 49, 1375-1385 (1953).

61. Johnson, J. C., Nozik, A. J. \& Michl, J. High triplet yield from singlet fission in a thin film of 1, 3-diphenylisobenzofuran. J. Am. Chem. Soc. 132, 16302-16303 (2010).

62. Ryerson, J. L. et al. Two thin film polymorphs of the singlet fission compound 1, 3-diphenylisobenzofuran. J. Phys. Chem. C 118, 12121-12132 (2014).

63. Schrauben, J. N., Ryerson, J. L., Michl, J. \& Johnson, J. C. Mechanism of singlet fission in thin films of 1, 3-diphenylisobenzofuran. J. Am. Chem. Soc. 136, 73637373 (2014).

64. Schwerin, A. F. et al. Toward designed singlet fission: electronic states and photophysics of 1, 3-diphenylisobenzofuran. J. Phys. Chem. A 114, 1457-1473 (2009).

65. Johnson, J. C. \& Michl, J. 1, 3-Diphenylisobenzofuran: a Model Chromophore for Singlet Fission. in Physical Organic Chemistry of Quinodimethanes 249-277 (Springer, 2017).

66. Minami, T. \& Nakano, M. Diradical character view of singlet fission. J. Phys. Chem. Lett. 3, 145-150 (2011).

67. Ito, S. \& Nakano, M. Theoretical Molecular Design of Heteroacenes for Singlet Fission: Tuning the Diradical Character by Modifying $\pi$-Conjugation Length and Aromaticity. J. Phys. Chem. C 119, 148-157 (2015).

68. Ito, S., Nagami, T. \& Nakano, M. Diradical Character-Based Design for Singlet Fission of Bisanthene Derivatives: Aromatic-Ring Attachment and $\pi$-Plane Twisting. J. Phys. Chem. Lett. 7, 3925-3930 (2016).

69. Akdag, A., Havlas, Z. \& Michl, J. Search for a small chromophore with efficient singlet fission: biradicaloid heterocycles. J. Am. Chem. Soc. 134, 14624-14631 (2012).

70. Viehe, H. G., Janousek, Z., Merenyi, R. \& Stella, L. The captodative effect. Acc. Chem. Res. 18, 148-154 (1985).

71. Zeng, T., Ananth, N. \& Hoffmann, R. Seeking small molecules for singlet fission: a heteroatom substitution strategy. J. Am. Chem. Soc. 136, 12638-12647 (2014).

72. Campbell, P. G., Marwitz, A. J. V \& Liu, S. Recent advances in azaborine chemistry. Angew. Chemie Int. Ed. 51, 6074-6092 (2012).

73. Wen, J., Havlas, Z. Z. \& Michl, J. Captodatively stabilized biradicaloids as chromophores for singlet fission. J. Am. Chem. Soc. 137, 165-172 (2014).

74. Bhattacharyya, K., Pratik, S. M. \& Datta, A. Small organic molecules for efficient singlet fission: role of silicon substitution. J. Phys. Chem. C 119, 25696-25702 
(2015).

75. Würfel, P. \& Würfel, U. Physics of solar cells: from basic principles to advanced concepts. (John Wiley \& Sons, 2016).

76. Zeng, T., Hoffmann, R. \& Ananth, N. The low-lying electronic states of pentacene and their roles in singlet fission. J. Am. Chem. Soc. 136, 5755-5764 (2014).

77. Minami, T., Ito, S. \& Nakano, M. Fundamental of Diradical-Character-Based Molecular Design for Singlet Fission. J. Phys. Chem. Lett. 4, 2133-2137 (2013).

78. Ito, S., Nagami, T. \& Nakano, M. Molecular design for efficient singlet fission. $J$. Photochem. Photobiol. C Photochem. Rev. 34, 85-120 (2018).

79. Japahuge, A. \& Zeng, T. Theoretical Studies of Singlet Fission: Searching for Materials and Exploring Mechanisms. Chempluschem 83, 146-182 (2018).

80. Zeng, T. \& Goel, P. Design of Small Intramolecular Singlet Fission Chromophores: An Azaborine Candidate and General Small Size Effects. J. Phys. Chem. Lett. 7, 1351-1358 (2016).

81. Zeng, T. Through-Linker Intramolecular Singlet Fission: General Mechanism and Designing Small Chromophores. J. Phys. Chem. Lett. 7, 4405-4412 (2016).

82. Zeng, T. et al. Identifying (BN)2-pyrenes as a New Class of Singlet Fission Chromophores: Significance of Azaborine Substitution. J. Phys. Chem. Lett. 9, 2919-2927 (2018).

83. Messelberger, J., Grünwald, A., Pinter, P., Hansmann, M. M. \& Munz, D. Carbene derived diradicaloids - building blocks for singlet fission? Chem. Sci. 9, 61076117 (2018).

84. Soleilhavoup, M. \& Bertrand, G. Cyclic (Alkyl)(Amino)Carbenes (CAACs): Stable Carbenes on the Rise. Acc. Chem. Res. 48, 256-266 (2015).

85. Hansmann, M. M., Melaimi, M., Munz, D. \& Bertrand, G. Modular Approach to Kekulé Diradicaloids Derived from Cyclic (Alkyl)(amino)carbenes. J. Am. Chem. Soc. 140, 2546-2554 (2018).

86. Kinjo, R., Donnadieu, B. \& Bertrand, G. Isolation of a Carbene-Stabilized Phosphorus Mononitride and Its Radical Cation ( $\mathrm{PN}+$.). Angew. Chemie Int. Ed. 49, 5930-5933 (2010).

87. Back, O., Donnadieu, B., Parameswaran, P., Frenking, G. \& Bertrand, G. Isolation of crystalline carbene-stabilized P2-radical cations and P2-dications. Nat. Chem. 2, 369 (2010).

88. Back, O. et al. A Crystalline Phosphinyl Radical Cation. J. Am. Chem. Soc. 132, 10262-10263 (2010).

89. Kinjo, R., Donnadieu, B., Celik, M. A., Frenking, G. \& Bertrand, G. Synthesis and Characterization of a Neutral Tricoordinate Organoboron Isoelectronic with Amines. Science (80-. ). 333, 610 LP - 613 (2011).

90. Lavallo, V., Canac, Y., Präsang, C., Donnadieu, B. \& Bertrand, G. Stable cyclic (alkyl)(amino)carbenes as rigid or flexible, bulky, electron-rich ligands for transition-metal catalysts: a quaternary carbon atom makes the difference. Angew. Chem. Int. Ed. Engl. 44, 5705-5709 (2005).

91. Pasto, D. J. Radical stabilization energies of disubstituted methyl radicals. A detailed theoretical analysis of the captodative effect. J. Am. Chem. Soc. 110, 8164-8175 (1988).

92. Lee, S. S., Filatov, M., Lee, S. S. \& Choi, C. H. Eliminating spin-contamination of 
spin-flip time dependent density functional theory within linear response formalism by the use of zeroth-order mixed-reference (MR) reduced density matrix. J. Chem. Phys. 149, 104101 (2018).

93. Lee, S. S. et al. Efficient implementations of analytic energy gradient for mixedreference spin-flip time-dependent density functional theory (MRSF-TDDFT). $J$. Chem. Phys. 150, 184111 (2019).

94. Lee, C., Yang, W. \& Parr, R. G. Development of the Colle-Salvetti correlationenergy formula into a functional of the electron density. Phys. Rev. B 37, 785-789 (1988).

95. Becke, A. D. Density-functional thermochemistry. III. The role of exact exchange. J. Chem. Phys. 98, 5648-5652 (1993).

96. Weigend, F. \& Ahlrichs, R. Balanced basis sets of split valence, triple zeta valence and quadruple zeta valence quality for $\mathrm{H}$ to $\mathrm{Rn}$ : Design and assessment of accuracy. Phys. Chem. Chem. Phys. 7, 3297-3305 (2005).

97. Schmidt, M. W. et al. General atomic and molecular electronic structure system. $J$. Comput. Chem. 14, 1347-1363 (1993).

98. Gordon, M. S. \& Schmidt, M. W. Chapter 41 - Advances in electronic structure theory: GAMESS a decade later. in (eds. Dykstra, C. E., Frenking, G., Kim, K. S. \& Scuseria, G. E. B. T.-T. and A. of C. C.) 1167-1189 (Elsevier, 2005). doi:https://doi.org/10.1016/B978-044451719-7/50084-6

99. Brédas, J.-L., Beljonne, D., Coropceanu, V. \& Cornil, J. Charge-Transfer and Energy-Transfer Processes in $\pi$-Conjugated Oligomers and Polymers: A Molecular Picture. Chem. Rev. 104, 4971-5004 (2004).

100. Volkhard, M. \& Oliver, K. No Title. (2003). doi:doi:10.1002/9783527602575

101. Navarro, A. et al. Effect of five-membered ring and heteroatom substitution on charge transport properties of perylene discotic derivatives: A theoretical approach. J. Chem. Phys. 145, 54903 (2016).

102. Angeli, C., Cimiraglia, R., Evangelisti, S., Leininger, T. \& Malrieu, J.-P. Introduction of n-electron valence states for multireference perturbation theory. $J$. Chem. Phys. 114, 10252-10264 (2001).

103. Angeli, C., Cimiraglia, R. \& Malrieu, J.-P. n-electron valence state perturbation theory: A spinless formulation and an efficient implementation of the strongly contracted and of the partially contracted variants. J. Chem. Phys. 117, 9138-9153 (2002).

104. Neese, F. The ORCA program system. Wiley Interdiscip. Rev. Comput. Mol. Sci. 2, 73-78 (2012).

105. Mayer, I. Bond order and valence: Relations to Mulliken's population analysis. Int. J. Quantum Chem. 26, 151-154 (1984).

106. Hoffmann, R. Interaction of orbitals through space and through bonds. Acc. Chem. Res. 4, 1-9 (1971).

107. Ozimiński, W. P. \& Dobrowolski, J. C. $\sigma$ - and $\pi$-electron contributions to the substituent effect: natural population analysis. J. Phys. Org. Chem. 22, 769-778 (2009).

108. Rahm, M., Zeng, T. \& Hoffmann, R. Electronegativity Seen as the Ground-State Average Valence Electron Binding Energy. J. Am. Chem. Soc. 141, 342-351 (2018). 
109. True, J. E., Thomas, T. D., Winter, R. W. \& Gard, G. L. Electronegativities from core-ionization energies: electronegativities of SF5 and CF3. Inorg. Chem. 42, 4437-4441 (2003).

110. Jin, L., Melaimi, M., Liu, L. \& Bertrand, G. Singlet carbenes as mimics for transition metals: synthesis of an air stable organic mixed valence compound [M 2 (C 2) ${ }^{\circ} ; \mathrm{M}=$ cyclic (alkyl)(amino) carbene]. Org. Chem. Front. 1, 351-354 (2014).

111. Li, Y. et al. C4 Cumulene and the Corresponding Air-Stable Radical Cation and Dication. Angew. Chemie Int. Ed. 53, 4168-4172 (2014).

112. Flynn, C. R. \& Michl, J. . pi.,. pi.-Biradicaloid hydrocarbons. o-Xylylene. Photochemical preparation from 1, 4-dihydrophthalazine in rigid glass, electric spectroscopy, and calculations. J. Am. Chem. Soc. 96, 3280-3288 (1974).

113. Marcus, R. A. Electron transfer reactions in chemistry. Theory and experiment. Rev. Mod. Phys. 65, 599 (1993).

114. Shaik, S. S. \& Hiberty, P. C. A chemist's guide to valence bond theory. (John Wiley \& Sons, 2007).

115. Prakash, G. K. S. et al. On the Nature of $\mathrm{C} \square \mathrm{H} \cdots \mathrm{F} \square \mathrm{C}$ Interactions in Hindered CF3 $\square$ C (sp3) Bond Rotations. Angew. Chemie Int. Ed. 50, 11761-11764 (2011).

116. Schwoerer, M. \& Wolf, H. C. Organic molecular solids. (John Wiley \& Sons, 2007). 